\title{
HETEROGENEOUS MULTISCALE FINITE ELEMENT METHOD WITH NOVEL NUMERICAL INTEGRATION SCHEMES*
}

\author{
RUI DU ${ }^{\dagger}$ AND PINGBING MING
}

\begin{abstract}
In this paper we introduce two novel numerical integration schemes within the framework of the heterogeneous multiscale method (HMM), when the finite element method is used as the macroscopic solver, to resolve the elliptic problem with a multiscale coefficient. For nonself-adjoint elliptic problems, optimal convergence rate is proved for the proposed methods, which naturally yields a new strategy for refining the macro-micro meshes and a criterion for determining the size of the microcell. Numerical results following this strategy show that the new methods significantly reduce the computational cost without loss of accuracy.
\end{abstract}

Key words. Heterogeneous multiscale method, finite element method, numerical integration schemes, elliptic homogenization problems.

AMS subject classifications. 65N30, 74Q05, 74Q15, 74Q20, 39A12.

\section{Introduction}

Consider the following elliptic problem:

$$
\left\{\begin{aligned}
-\nabla \cdot\left(a^{\varepsilon}(\boldsymbol{x}) \nabla u^{\varepsilon}(\boldsymbol{x})\right) & =f(\boldsymbol{x}) & & \text { in } D \subset \mathbb{R}^{d}, \\
u^{\varepsilon}(\boldsymbol{x}) & =0 & & \text { on } \partial D,
\end{aligned}\right.
$$

where $\varepsilon$ is a small parameter that signifies the multiscale nature of the problem. The matrix $a^{\varepsilon}(\boldsymbol{x})=\left(a_{i j}^{\varepsilon}(\boldsymbol{x})\right)_{i, j=1}^{d}$ is assumed to have bounded measurable entries, i.e., $\max _{i, j}\left\|a_{i j}^{\varepsilon}\right\|_{L^{\infty}(D)}=\Lambda<\infty$, and satisfies the uniform ellipticity condition: for $\boldsymbol{\xi} \in \mathbb{R}^{d}$,

$$
\lambda \sum_{i=1}^{d} \xi_{i}^{2} \leq \sum_{i, j=1}^{d} a_{i j}^{\varepsilon} \xi_{i} \xi_{j}
$$

with $\lambda>0$, but the matrix $a^{\varepsilon}$ is not necessarily symmetric. This problem (1.1) plays a fundamental and practical role in several fields that include mechanical properties of composite materials and flow transport in porous media for example.

In the sense of H-convergence (see [27]), for every $f \in H^{-1}(D)$ the sequence $\left\{u^{\varepsilon}\right\}$ of solutions of (1.1) satisfies

$$
\left\{\begin{array}{ccc}
u^{\varepsilon} \rightarrow U_{0} & \text { weakly in } & H_{0}^{1}(D), \\
a^{\varepsilon} \nabla u^{\varepsilon} \rightarrow \mathcal{A} \nabla U_{0} & \text { weakly in } & \left(L^{2}(D)\right)^{d},
\end{array}\right.
$$

where $U_{0}$ is the solution of the problem

$$
\left\{\begin{aligned}
-\nabla \cdot\left(\mathcal{A}(\boldsymbol{x}) \nabla U_{0}(\boldsymbol{x})\right) & =f(\boldsymbol{x}) & & \text { in } D, \\
U_{0}(\boldsymbol{x}) & =0 & & \text { on } \partial D .
\end{aligned}\right.
$$

*Received: September 3, 2009; accepted (in revised version): November 29, 2009. Communicated by Weinan E.

$\dagger$ Institute of Computational Mathematics and Scientific/Engineering Computing, AMSS, Chinese Academy of Sciences, Beijing, 100190, People's Republic of China (durui@lsec.cc.ac.cn).

${ }^{\ddagger}$ LSEC, Institute of Computational Mathematics and Scientific/Engineering Computing, AMSS, Chinese Academy of Sciences, Beijing, 100190, People's Republic of China (mpb@lsec.cc.ac.cn). http://lsec.cc.ac.cn/ ${ }^{\sim}$ mpb. The work of Ming was supported in part by National Natural Science Foundation of China grants 10871197 and 10932011, and National Basic Research Program grant 2005 CB321704. 
In general, there is no explicit formula for the effective matrix $\mathcal{A}$ except in one dimensional problems [27].

The heterogeneous multiscale method introduced in [15] is a general methodology for designing and analyzing multiscale methods. It consists of two ingredients: a macroscopic scheme for macrovariables on a macrogrid and estimating the missing macroscopic data from the microscopic model. The efficiency of HMM lies in the following two points: 1) its flexibility and 2) the ability to extract the missing macroscale data from microscale models with minimum cost, by exploiting scale separation or other special features of the problems in study. In HMM framework, the finite element method can be readily employed as the macroscopic solver. The so-called heterogeneous multiscale finite element method (HMM-FEM) has been developed to solve (1.1); see [15, 16, 23, 28, 1, 4] and references therein. In HMM-FEM, the missing macroscopic data is the effective matrix, which is obtained by solving the microcell problems posed at the quadrature nodes employed in finite element method.

In practice, solving the cell problems numerically is the main computational cost of HMM-FEM [22, 1], while the number of the cell problems is $d$ times of that of the macro quadrature nodes. Therefore, if we can reduce the number of the quadrature nodes, the cost is decreased; even if the cell problems are computed in parallel, the number of processors is reduced. Based on this observation, we propose two strategies to reduce the number of cell problems without loss of accuracy. First, we introduce two new numerical integration schemes which have fewer quadrature nodes in total compared to the conventional numerical integration schemes in [16]. The new schemes employ either the vertices of elements or points over the common edge of two adjacent elements as the quadrature nodes. Second, we use a quadratic finite element method instead of a linear finite element method as the macroscopic solver. In this way, we need to solve fewer cell problems to obtain the same accuracy provided the solution is smooth enough. This is confirmed by the results in section 3 and section 4 . Though using high order finite element methods for the macroscopic solver in HMM-FEM was introduced and analyzed in [16], their numerical performance has not been evaluated to date. The advantage of a higher-order macroscopic solver has been demonstrated in [10] when discontinuous Galerkin method is employed as the macroscopic solver in the HMM framework, though not in HMM-FEM.

Besides reduction of the number of the cell problems, an alternative approach to reduce the cost is to avoid over-resolving the cell problems. Based on the optimal error estimate of the proposed method, we present a strategy for refining the macro-micro meshes as well as a criterion for determining the size of the microcell. Following this principle, the cell problems with varied size are solved numerically with minimum accuracy that may be sufficient for achieving the desired overall accuracy. Therefore, the computational cost is significantly reduced. A refinement strategy for the macromicro meshes was proposed in $[3,2]$, which works for the cell problem with fixed cell size.

The cell problems in HMM-FEM are usually approximated by linear finite elements [22]. The discretization error was carefully studied in [1] for the elliptic homogenization problem with periodic coefficients. Using a pseudo-spectral method as the microscale solver was investigated in [4] to achieve nearly optimal computing complexity. In both works, the cell problems are posed over a cell whose size is equal to the exact period and supplemented with periodic boundary condition. The analysis essentially relies on the explicit expression of the solutions of the cell problems; see $[1,(3.14)]$ and $[4,(3.23)]$. Such explicit expression is only available when the cell size 
is an integer multiple of the period. However, the exact period is usually unknown due to the uncertainty of the input data $[13,8]$. This means that the cell problems are usually posed over a cell whose size is not necessarily an integer time of the period, and hence we cannot expect the explicit expression of the solutions of the cell problems in general. In this work we solve the cell problems with Dirichlet boundary condition and the cell size is not an integer time of the period. Our analysis is based on a new regularity result proved in the Appendix, and it covers the case when $a^{\varepsilon}$ is non-symmetric. The author in [2] studied the same case, while the error estimate therein only applies to the case when $a^{\varepsilon}$ is symmetric and under certain unjustified smoothness assumptions on the solutions of the cell problems.

Following the framework established in [16], we prove the optimal error estimate of the proposed method, which is also confirmed by extensive numerical examples. Observe that the analysis in [16] essentially depends on the assumption that $a^{\varepsilon}$ is a symmetric matrix, while our analysis covers the case when $a^{\varepsilon}$ is non-symmetric. This is achieved by resorting to the adjoint problem used in H-convergence theory of [27].

The paper is organized as follows. In section 2, we introduce a HMM-FEM based on two new numerical integration schemes. An error estimate of this method is proven in section 3. Numerical results are reported in section 4 and conclusions are drawn in the last section.

Most results in this paper apply to $d$ dimensional problems. However, we focus on the $2-\mathrm{d}$ case for brevity of exposition.

Throughout this paper we use the standard notation $H^{k}(D)$ for a Sobolev space of order $k$ with norm given by $\|\cdot\|_{H^{k}(D)}$; see e.g., [6] for exact definition. For $k=0$ we write $L^{2}(D)$ instead of $H^{0}(D)$ and denote the norm and inner product by $\|\cdot\|_{L^{2}(D)}$ and $(\cdot, \cdot)$, respectively. The standard Euclidean norm will be denoted by $\|\cdot\|$.

The generic constant $C$ is assumed to be independent of the parameters $\varepsilon, \delta, H$ and $h$, whose meaning will be specified later.

\section{HMM-FEM with novel numerical integration schemes}

The macroscopic solver is chosen as the standard $P_{k}$ element over a triangulation $\mathcal{T}_{H}$ of mesh size $H$, and the corresponding finite element space is denoted by $X_{H}$ [11]. The HMM solution $U_{H} \in X_{H}$ satisfies

$$
A_{H}\left(U_{H}, V\right)=(f, V) \text { for all } V \in X_{H},
$$

where the bilinear form $A_{H}$ is defined for any $V, W \in X_{H}$ by

$$
A_{H}(V, W) \equiv \sum_{K \in \mathcal{T}_{H}}|K| \sum_{\ell=1}^{L} \omega_{\ell} f_{I_{\delta}\left(\boldsymbol{x}_{\ell}\right)} \nabla v_{h}^{\varepsilon}(\boldsymbol{x}) \cdot a^{\varepsilon}(\boldsymbol{x}) \nabla w_{h}^{\varepsilon}(\boldsymbol{x}) \mathrm{d} \boldsymbol{x},
$$

where $\left\{\boldsymbol{x}_{\ell}\right\}_{\ell=1}^{L}$ and $\left\{\omega_{\ell}\right\}_{\ell=1}^{L}$ are the quadrature nodes and weights in $K,|K|$ is the volume of $K$, and the cell $I_{\delta}\left(\boldsymbol{x}_{\ell}\right)$ is a square of size $\delta$ centered at $\boldsymbol{x}_{\ell}$. Moreover, we may replace $I_{\delta}\left(\boldsymbol{x}_{\ell}\right)$ by $I_{\delta}$ when no confusion occurs. The function $w_{h}^{\varepsilon}$ is defined as the solution of the following cell problem: $w_{h}^{\varepsilon}-W_{\ell} \in X_{h}$ satisfies

$$
\int_{I_{\delta}\left(\boldsymbol{x}_{\ell}\right)} \nabla z \cdot a^{\varepsilon}(\boldsymbol{x}) \nabla w_{h}^{\varepsilon} \mathrm{d} \boldsymbol{x}=0 \quad \text { for all } z \in X_{h},
$$

where $W_{\ell} \equiv W\left(\boldsymbol{x}_{\ell}\right)+\left(\boldsymbol{x}-\boldsymbol{x}_{\ell}\right) \cdot \nabla W\left(\boldsymbol{x}_{\ell}\right)$ is the linear approximation of $W$ at $\boldsymbol{x}_{\ell}$. The microscopic finite element space $X_{h}$ is defined by

$$
X_{h} \equiv\left\{v \in H_{0}^{1}\left(I_{\delta}\left(\boldsymbol{x}_{\ell}\right)\right)|v|_{K} \in P_{1}(K), K \in \mathcal{T}_{h}\right\},
$$


where $\mathcal{T}_{h}$ is the triangulation of $I_{\delta}\left(\boldsymbol{x}_{\ell}\right)$ of mesh size $h$. The function $v_{h}^{\varepsilon}$ is defined in the same manner as $w_{h}^{\varepsilon}$ with $W$ replaced by $V \in X_{H}$.

According to $[15,16]$, the bilinear form $A_{H}$ can be understood as follows. For any $V, W \in X_{H}$, at each $\boldsymbol{x}_{\ell}$, if we define a matrix $\mathcal{A}_{H}$ by

$$
\left(\nabla V \cdot \mathcal{A}_{H} \nabla W\right)\left(\boldsymbol{x}_{\ell}\right) \equiv f_{I_{\delta}\left(\boldsymbol{x}_{\ell}\right)} \nabla v_{h}^{\varepsilon}(\boldsymbol{x}) \cdot a^{\varepsilon}(\boldsymbol{x}) \nabla w_{h}^{\varepsilon}(\boldsymbol{x}) \mathrm{d} \boldsymbol{x}
$$

then the bilinear form can be rewritten as

$$
A_{H}(V, W)=\sum_{K \in \mathcal{T}_{H}} Q_{K}\left(\nabla V \cdot \mathcal{A}_{H} \nabla W\right)
$$

where $Q_{K}$ is a quadrature scheme that approximates $\int_{K} p$ for any function $p$ as

$$
Q_{K}(p)=|K| \sum_{\ell=1}^{L} \omega_{\ell} p\left(\boldsymbol{x}_{\ell}\right) \quad \text { with } \quad \omega_{\ell}>0, \ell=1, \ldots, L .
$$

The following condition for $Q_{K}$ has been introduced in [16] to ensure the accuracy of HMM-FEM:

$$
Q_{K}(p)= \begin{cases}\int_{K} p(\boldsymbol{x}) \mathrm{d} \boldsymbol{x} & \text { for all } p \in P_{2 k-2}, k \geq 2, \\ \int_{K} p(\boldsymbol{x}) \mathrm{d} \boldsymbol{x} & \text { for all } p \in P_{1} .\end{cases}
$$

This condition immediately implies

$$
Q_{K}\left(|\nabla V|^{2}\right)=\int_{K}|\nabla V|^{2} \mathrm{~d} \boldsymbol{x} \quad \text { for all } K \in \mathcal{T}_{H}, V \in X_{H}
$$

Numerical integration schemes satisfying (2.5) can be found in [26]. We list some schemes for $k=1,2$ by specifying the quadrature nodes and the weights, and refer to figure 2.1 for the location of the quadrature nodes.

1. Vertices-based integration scheme:

$$
\left\{\omega_{\ell}\right\}_{\ell=1}^{3}=\frac{1}{3}, \quad\left\{\boldsymbol{x}_{\ell}\right\}_{\ell=1}^{3} \text { are the vertices of } K .
$$

2. Barycenter-based integration scheme:

$$
\omega=1, \quad \boldsymbol{x} \text { is the barycenter of } K .
$$

3. Edge-based integration scheme:

$$
\left\{\omega_{\ell}\right\}_{\ell=1}^{3}=\frac{1}{3}, \quad\left\{\boldsymbol{x}_{\ell}\right\}_{\ell=1}^{3} \text { are the middle points of each edge of } K .
$$

4. Element-based integration scheme:

$$
\begin{aligned}
\omega_{1} & =\omega_{2}=\omega_{3}=\frac{1}{3} \\
\boldsymbol{x}_{1}=(2 / 3,1 / 6,1 / 6), \quad \boldsymbol{x}_{2} & =(1 / 6,2 / 3,1 / 6), \quad \boldsymbol{x}_{3}=(1 / 6,1 / 6,2 / 3)
\end{aligned}
$$

in the sense of barycentric coordinates [11]. 

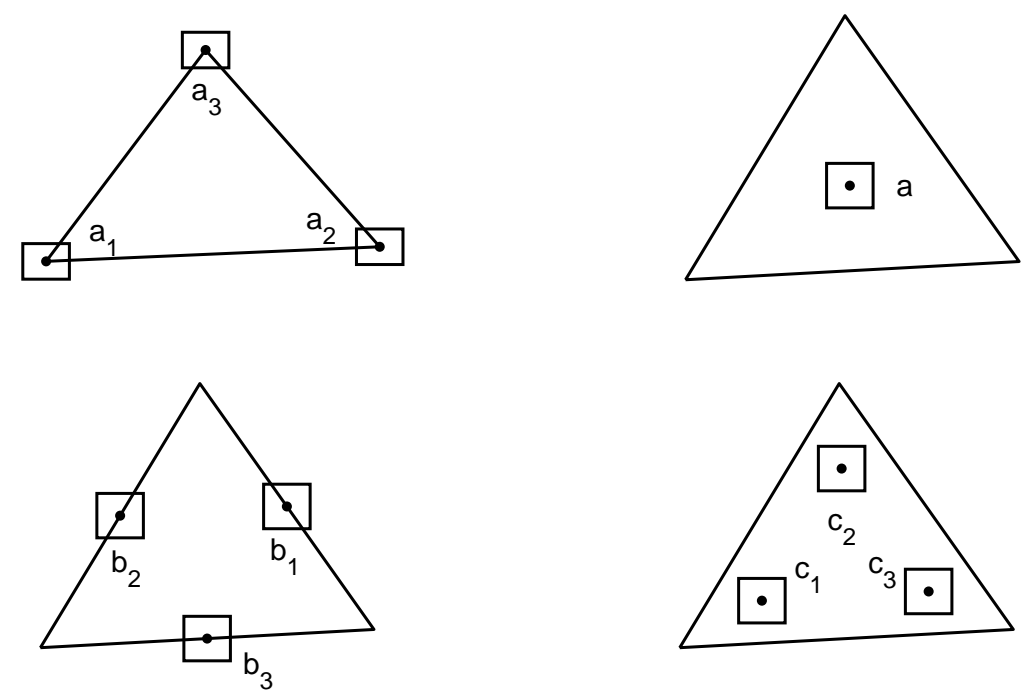

Figure 2.1. The dots are the quadrature nodes. The little squares are the microcells $I_{\delta}$ centered at the quadrature nodes. Top left: scheme (2.7), top right: scheme (2.8); bottom left: scheme (2.9), bottom right: scheme (2.10).

It is straightforward to verify that the above four schemes satisfy (2.5). Therefore, all of them can be readily used in HMM-FEM. Indeed, the scheme (2.10) has appeared in [16]. The schemes (2.8) and (2.9) are frequently used in practice, while the schemes (2.7) and (2.10) introduced in [17, Theorem 2] seem rarely used. However, HMM-FEM based on (2.7) and (2.9) significantly reduce the cost without loss of accuracy compared with the methods based on (2.8) and (2.10), respectively. This observation is the main motivation of the present investigation and will be confirmed by the theoretical and numerical results in the following two sections.

The cell problem (2.3) is supplemented with Dirichlet boundary condition. It may be replaced by other boundary conditions such as periodic boundary condition (see section 3) and Neumann boundary condition. Different boundary conditions have pros and cons in practice. We refer to [28] for a systematic study of the influence of various boundary conditions on the accuracy and efficiency of HMM-FEM. See also [25] for related discussion from the mechanics point of view.

\section{Error estimate of HMM-FEM}

We first prove the following lemma that implies the existence and uniqueness of the proposed methods. The proof essentially follows the same way of [16, Lemma 1.9] with a minor modification since the matrix $a^{\varepsilon}$ is not necessarily symmetric.

Lemma 3.1. Let $A_{H}$ be defined in (2.2). Then

$$
A_{H}(V, V) \geq \lambda\|\nabla V\|_{L^{2}(D)}^{2} \quad \text { for all } V \in X_{H},
$$

and

$$
\left|A_{H}(V, W)\right| \leq \frac{\Lambda^{2}}{\lambda}\|\nabla V\|_{L^{2}(D)}\|\nabla W\|_{L^{2}(D)} \quad \text { for all } V, W \in X_{H} .
$$


Proof. Noticing that $v_{h}^{\varepsilon}=V_{\ell}$ on $\partial I_{\delta}\left(\boldsymbol{x}_{\ell}\right)$ and $\nabla V_{\ell}$ is a constant vector in $I_{\delta}\left(\boldsymbol{x}_{\ell}\right)$, we obtain

$$
\int_{I_{\delta}\left(\boldsymbol{x}_{\ell}\right)} \nabla V_{\ell} \cdot \nabla\left(v_{h}^{\varepsilon}-V_{\ell}\right) \mathrm{d} \boldsymbol{x}=0
$$

which immediately implies

$$
\left\|\nabla v_{h}^{\varepsilon}\right\|_{L^{2}\left(I_{\delta}\right)}^{2}=\left\|\nabla\left(v_{h}^{\varepsilon}-V_{\ell}\right)\right\|_{L^{2}\left(I_{\delta}\right)}^{2}+\left\|\nabla V_{\ell}\right\|_{L^{2}\left(I_{\delta}\right)}^{2} .
$$

Using the ellipticity of $a^{\varepsilon}$, the above identity, and (2.6), we obtain

$$
\begin{aligned}
A_{H}(V, V) & \geq \lambda \sum_{K \in \mathcal{T}_{H}} \frac{|K|}{\left|I_{\delta}\right|} \sum_{\ell=1}^{L} \omega_{\ell}\left\|\nabla v_{h}^{\varepsilon}\right\|_{L^{2}\left(I_{\delta}\right)}^{2} \geq \lambda \sum_{K \in \mathcal{T}_{H}} \frac{|K|}{\left|I_{\delta}\right|} \sum_{\ell=1}^{L} \omega_{\ell}\left\|\nabla V_{\ell}\right\|_{L^{2}\left(I_{\delta}\right)}^{2} \\
& =\lambda \sum_{K \in \mathcal{T}_{H}}|K| \sum_{\ell=1}^{L} \omega_{\ell}\left|\nabla V\left(\boldsymbol{x}_{\ell}\right)\right|^{2}=\lambda \sum_{K \in \mathcal{T}_{H}} Q_{K}\left(|\nabla V|^{2}\right)=\lambda\|\nabla V\|_{L^{2}(D)}^{2} .
\end{aligned}
$$

This gives (3.1).

Taking $z=w_{h}^{\varepsilon}-W_{\ell}$ in (2.3), we obtain

$$
\int_{I_{\delta}\left(\boldsymbol{x}_{\ell}\right)} \nabla w_{h}^{\varepsilon} \cdot a^{\varepsilon}(\boldsymbol{x}) \nabla w_{h}^{\varepsilon} \mathrm{d} \boldsymbol{x}=\int_{I_{\delta}\left(\boldsymbol{x}_{\ell}\right)} \nabla W_{\ell} \cdot a^{\varepsilon}(\boldsymbol{x}) \nabla w_{h}^{\varepsilon} \mathrm{d} \boldsymbol{x},
$$

which immediately implies

$$
\left\|\nabla w_{h}^{\varepsilon}\right\|_{L^{2}\left(I_{\delta}\right)} \leq \frac{\Lambda}{\lambda}\left\|\nabla W_{\ell}\right\|_{L^{2}\left(I_{\delta}\right)} .
$$

Taking $z=v_{h}^{\varepsilon}-V_{\ell}$ in (2.3), we write $A_{H}$ for any $V, W \in X_{H}$ as

$$
A_{H}(V, W)=\sum_{K \in \mathcal{T}_{H}} \frac{|K|}{\left|I_{\delta}\right|} \sum_{\ell=1}^{L} \omega_{\ell} \int_{I_{\delta}\left(\boldsymbol{x}_{\ell}\right)} \nabla V_{\ell} \cdot a^{\varepsilon}(\boldsymbol{x}) \nabla w_{h}^{\varepsilon} \mathrm{d} \boldsymbol{x} .
$$

Substituting the estimate (3.3) into the above equation and invoking (2.6) once again, we obtain

$$
\begin{aligned}
\left|A_{H}(V, W)\right| & \leq \frac{\Lambda^{2}}{\lambda} \sum_{K \in \mathcal{T}_{H}} \frac{|K|}{\left|I_{\delta}\right|} \sum_{\ell=1}^{L} \omega_{\ell}\left\|\nabla V_{\ell}\right\|_{L^{2}\left(I_{\delta}\right)}\left\|\nabla W_{\ell}\right\|_{L^{2}\left(I_{\delta}\right)} \\
& =\frac{\Lambda^{2}}{\lambda} \sum_{K \in \mathcal{T}_{H}}|K| \sum_{\ell=1}^{L} \omega_{\ell}\left|\nabla V\left(\boldsymbol{x}_{\ell}\right)\right|\left|\nabla W\left(\boldsymbol{x}_{\ell}\right)\right| \\
& \leq \frac{\Lambda^{2}}{\lambda} \sum_{K \in \mathcal{T}_{H}} Q_{K}^{1 / 2}\left(|\nabla V|^{2}\right) Q_{K}^{1 / 2}\left(|\nabla W|^{2}\right) \\
& =\frac{\Lambda^{2}}{\lambda} \sum_{K \in \mathcal{T}_{H}}\|\nabla V\|_{L^{2}(K)}\|\nabla W\|_{L^{2}(K)} \\
& \leq \frac{\Lambda^{2}}{\lambda}\|\nabla V\|_{L^{2}(D)}\|\nabla W\|_{L^{2}(D)},
\end{aligned}
$$


which gives (3.2).

A direct consequence of the estimates (3.1) and (3.2) is, for $\boldsymbol{\xi} \in \mathbb{R}^{2}$,

$$
\lambda\left(\xi_{1}^{2}+\xi_{2}^{2}\right) \leq \sum_{i, j=1}^{2}\left(\mathcal{A}_{H}\right)_{i j}\left(\boldsymbol{x}_{\ell}\right) \xi_{i} \xi_{j} \leq \frac{\Lambda^{2}}{\lambda}\left(\xi_{1}^{2}+\xi_{2}^{2}\right),
$$

which leads to the same lower and upper bounds as the H-limit of the effective matrix $\mathcal{A}$ at every quadrature node $\boldsymbol{x}_{\ell} ;$ see [27]. If the matrix $a^{\varepsilon}$ is symmetric, then the upper bound $\Lambda^{2} / \lambda$ is replaced by $\Lambda[16,27]$.

Based on the above lemma, as in [16, Theorem 1.1], we obtain

Theorem 3.2. Let $U_{0}$ and $U_{H}$ be the solutions of (1.2) and (2.1), respectively. Define

$$
e(\mathrm{HMM})=\max _{\substack{K \in \mathcal{T}_{H} \\ \boldsymbol{x}_{\ell} \in K}}\left\|\left(\mathcal{A}-\mathcal{A}_{H}\right)\left(\boldsymbol{x}_{\ell}\right)\right\| .
$$

If $U_{0} \in H^{k+1}(D)$ and (2.5) holds, then there exists $C$ such that

$$
\begin{aligned}
\left\|U_{0}-U_{H}\right\|_{H^{1}(D)} & \leq C\left(H^{k}+e(\mathrm{HMM})\right), \\
\left\|U_{0}-U_{H}\right\|_{L^{2}(D)} & \leq C\left(H^{k+1}+e(\mathrm{HMM})\right) .
\end{aligned}
$$

So far no assumption on $a^{\varepsilon}$ is necessary except for its ellipticity and boundedness. Under such conditions the best result we know is $e(\mathrm{HMM}) \rightarrow 0$ if $\varepsilon \rightarrow 0$ first and $\delta \rightarrow 0, H \rightarrow 0$ next provided that $\mathcal{A}(\boldsymbol{x})$ is continuous; see [14, Lemma 1.4] for a proof. To get the exact convergence order of $e(\mathrm{HMM})$, we have to make further assumptions on the form of $a^{\varepsilon}$. In this paper, we assume that each entry of $a^{\varepsilon}$ is a locally periodic function, i.e., $a_{i j}^{\varepsilon}(\boldsymbol{x})=a_{i j}(\boldsymbol{x}, \boldsymbol{x} / \varepsilon)$ and $a_{i j}(\boldsymbol{x}, \boldsymbol{y})$ is periodic in $\boldsymbol{y}$ with period $Y=(-1 / 2,1 / 2)^{2}$ for $i, j=1,2$. We refer to [16] for the estimate of $e$ (HMM) when $a^{\varepsilon}$ is a random stationary field. by

For the problem with locally periodic coefficients, the effective matrix $\mathcal{A}$ is given

$$
\mathcal{A}_{i j}(\boldsymbol{x})=f_{Y}\left(a_{i j}+a_{i k} \frac{\partial \chi^{j}}{\partial y_{k}}\right)(\boldsymbol{x}, \boldsymbol{y}) \mathrm{d} \boldsymbol{y}
$$

where the functions $\boldsymbol{\chi}=\left\{\chi^{j}\right\}_{j=1}^{2}$ are periodic in $\boldsymbol{y}$ with period $Y$ and satisfy

$$
\left\{\begin{array}{c}
\frac{\partial}{\partial y_{i}}\left(a_{i k} \frac{\partial \chi^{j}}{\partial y_{k}}\right)(\boldsymbol{x}, \boldsymbol{y})=-\left(\frac{\partial}{\partial y_{i}} a_{i j}\right)(\boldsymbol{x}, \boldsymbol{y}) \text { in } Y, \\
\int_{Y} \chi^{j}(\boldsymbol{x}, \boldsymbol{y}) \mathrm{d} \boldsymbol{y}=0 .
\end{array}\right.
$$

This cell problem is solvable due to $(3.4)_{2}$ (see [9]). We assume that there exists $C$ such that for $j=1,2$, and $s \in \mathbb{N}$

$$
\left|D_{\boldsymbol{y}}^{\boldsymbol{\alpha}} \chi^{j}(\boldsymbol{x}, \boldsymbol{y})\right| \leq C \quad \text { for all } \boldsymbol{x} \in D \text { and } \boldsymbol{y} \in Y,|\boldsymbol{\alpha}| \leq s,
$$

where $\boldsymbol{\alpha}=\left(\alpha_{1}, \alpha_{2}\right)$ is a multi-index whose components $\alpha_{i}$ are non-negative integers, $|\boldsymbol{\alpha}|=\alpha_{1}+\alpha_{2}$, and $D_{\boldsymbol{y}}^{\boldsymbol{\alpha}}=\partial^{|\boldsymbol{\alpha}|} / \partial y_{1}^{\alpha_{1}} \partial y_{2}^{\alpha_{2}}$. 
TheOREm 3.3. If $a_{i j}(\boldsymbol{x}, \boldsymbol{y})$ is smooth in both $\boldsymbol{x}$ and $\boldsymbol{y}$ for $i, j=1,2$, and (3.5) is true for $s=1$, then

$$
e(\mathrm{HMM}) \leq C\left(\delta+\frac{\varepsilon}{\delta}+\frac{h^{2}}{\varepsilon^{2}}\right)
$$

REMARK 3.4. If $a^{\varepsilon}$ is strictly periodic, i.e., $a_{i j}^{\varepsilon}(\boldsymbol{x})=a_{i j}(\boldsymbol{x} / \varepsilon)$, and $a_{i j}(\boldsymbol{y})$ is a periodic function with period $Y$, or $a^{\varepsilon}$ is locally periodic and $a^{\varepsilon}=a(\boldsymbol{x}, \boldsymbol{x} / \varepsilon)$ is replaced by $a_{\ell}^{\varepsilon}=a\left(\boldsymbol{x}_{\ell}, \boldsymbol{x} / \varepsilon\right)$ in (2.3), then $\delta$ in the estimates (3.6) and (3.25) below may be dropped; see (3.22) and also [5].

In order to prove (3.6), we define an auxiliary matrix $\widetilde{\mathcal{A}}_{H}$ by

$$
\left(\nabla V \cdot \widetilde{\mathcal{A}}_{H} \nabla W\right)\left(\boldsymbol{x}_{\ell}\right)=f_{I_{\delta}\left(\boldsymbol{x}_{\ell}\right)} \nabla \widehat{v}^{\varepsilon} \cdot a_{\ell}^{\varepsilon} \nabla \widehat{w}^{\varepsilon} \mathrm{d} \boldsymbol{x} \text { for all } V, W \in X_{H},
$$

where $\widehat{w}^{\varepsilon}, \widehat{v}^{\varepsilon}$ are defined respectively as: find $\widehat{w}^{\varepsilon}-W_{\ell} \in H_{0}^{1}\left(I_{\delta}\right)$ such that

$$
\int_{I_{\delta}\left(\boldsymbol{x}_{\ell}\right)} \nabla z \cdot a_{\ell}^{\varepsilon} \nabla \widehat{w}^{\varepsilon} \mathrm{d} \boldsymbol{x}=0 \text { for all } z \in H_{0}^{1}\left(I_{\delta}\right),
$$

and find $\widehat{v}^{\varepsilon}-V_{\ell} \in H_{0}^{1}\left(I_{\delta}\right)$ such that

$$
\int_{I_{\delta}\left(\boldsymbol{x}_{\ell}\right)} \nabla z \cdot a_{\ell}^{\varepsilon} \nabla \widehat{v}^{\varepsilon} \mathrm{d} \boldsymbol{x}=0 \quad \text { for all } \quad z \in H_{0}^{1}\left(I_{\delta}\right) .
$$

We define $\widetilde{w}^{\varepsilon}$ as the solution of the adjoint problem of (3.7) with $a_{\ell}^{\varepsilon}$ replaced by its transpose $\left(a_{\ell}^{\varepsilon}\right)^{t}$, and define $\widehat{w}_{h}^{\varepsilon}, \widetilde{w}_{h}^{\varepsilon} \in X_{h}$ as the finite element approximation solutions of $\widehat{w}^{\varepsilon}$ and $\widetilde{w}^{\varepsilon}$, respectively. We also define $\widetilde{v}^{\varepsilon}, \widehat{v}_{h}^{\varepsilon}, \widetilde{v}_{h}^{\varepsilon}$ in the same manner as $\widetilde{w}^{\varepsilon}, \widehat{w}_{h}^{\varepsilon}, \widetilde{w}_{h}^{\varepsilon}$, respectively.

The first step is to bound $\left\|\left(\mathcal{A}-\widetilde{\mathcal{A}}_{H}\right)\left(\boldsymbol{x}_{\ell}\right)\right\|$ for any $\boldsymbol{x}_{\ell} \in K$.

Lemma 3.5. Under the same condition of Theorem 3.3, we have, for any $\boldsymbol{x}_{\ell} \in K$,

$$
\left\|\left(\mathcal{A}-\widetilde{\mathcal{A}}_{H}\right)\left(\boldsymbol{x}_{\ell}\right)\right\| \leq C \frac{\varepsilon}{\delta} .
$$

When $a^{\varepsilon}$ is symmetric, the above estimate was proved in [16, Theorem 1.2]. However, a direct application of the argument leads to a suboptimal estimate of $\|(\mathcal{A}-$ $\left.\widetilde{\mathcal{A}}_{H}\right)\left(\boldsymbol{x}_{\ell}\right) \|$, which seems to contradict the numerical results summarized in figure 4.3. We prove (3.9) by exploiting the adjoint problems of (3.7) and (3.8) and proceeding along the same lines of [23, Theorem 1.2], which is essentially the same with [16, Theorem 1.2].

Estimating $\left\|\left(\mathcal{A}-\widetilde{\mathcal{A}}_{H}\right)\left(\boldsymbol{x}_{\ell}\right)\right\|$ consists of two steps. First, we estimate $\|(\mathcal{A}-$ $\widehat{\mathcal{A}})\left(\boldsymbol{x}_{\ell}\right) \|$, where the matrix $\widehat{\mathcal{A}}\left(\boldsymbol{x}_{\ell}\right)$ is defined by

$$
(\nabla V \cdot \widehat{\mathcal{A}} \nabla W)\left(\boldsymbol{x}_{\ell}\right)=f_{I_{\delta}\left(\boldsymbol{x}_{\ell}\right)} \nabla \widehat{V}^{\varepsilon} \cdot a_{\ell}^{\varepsilon} \nabla \widehat{W}^{\varepsilon} \quad \text { for all } \quad V, W \in X_{H},
$$

where

$$
\widehat{V}^{\varepsilon}=V_{\ell}+\varepsilon\left(\boldsymbol{\chi}_{\ell} \cdot \nabla\right) V_{\ell} \text { and } \widehat{W}^{\varepsilon}=W_{\ell}+\varepsilon\left(\boldsymbol{\chi}_{\ell} \cdot \nabla\right) W_{\ell}
$$


with $\chi_{\ell}=\chi\left(\boldsymbol{x}_{\ell}, \boldsymbol{x} / \varepsilon\right)$.

Second, we estimate $\left\|\left(\widehat{\mathcal{A}}-\widetilde{\mathcal{A}}_{H}\right)\left(\boldsymbol{x}_{\ell}\right)\right\|$, which is based on the following identity:

$$
\begin{aligned}
& \left(\nabla V \cdot\left(\widetilde{\mathcal{A}}_{H}-\widehat{\mathcal{A}}\right) \nabla W\right)\left(\boldsymbol{x}_{\ell}\right) \\
& =f_{I_{\delta}\left(\boldsymbol{x}_{\ell}\right)}\left[\nabla \widehat{v}^{\varepsilon} \cdot a_{\ell}^{\varepsilon} \nabla\left(\widehat{w}^{\varepsilon}-\widehat{W}^{\varepsilon}\right)+\nabla\left(\widehat{v}^{\varepsilon}-\widehat{V}^{\varepsilon}\right) \cdot a_{\ell}^{\varepsilon} \nabla \widehat{W}^{\varepsilon}\right] \mathrm{d} \boldsymbol{x} .
\end{aligned}
$$

Finally, the estimate of $\left\|\left(\mathcal{A}-\widetilde{\mathcal{A}}_{H}\right)\left(\boldsymbol{x}_{\ell}\right)\right\|$ follows from the triangle inequality.

A straightforward calculation gives

$$
\nabla \cdot\left(a_{\ell}^{\varepsilon} \nabla \widehat{V}^{\varepsilon}\right)=0 \quad \text { and } \quad \nabla \cdot\left(a_{\ell}^{\varepsilon} \nabla \widehat{W}^{\varepsilon}\right)=0
$$

Define $\theta^{\varepsilon} \equiv \widehat{w}^{\varepsilon}-\widehat{W}^{\varepsilon}$, which satisfies

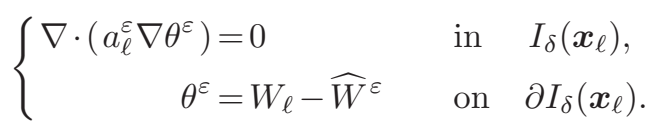

Lemma 3.6. Let $\theta^{\varepsilon}$ be the solution of (3.12). Then

$$
\left\|\nabla \theta^{\varepsilon}\right\|_{L^{2}\left(I_{\delta}\right)} \leq C\left(\frac{\varepsilon}{\delta}\right)^{1 / 2}\left\|\nabla W_{\ell}\right\|_{L^{2}\left(I_{\delta}\right)} .
$$

Proof. Denote by $I_{\kappa \varepsilon}=\boldsymbol{x}_{\ell}+\kappa \varepsilon Y$, where $\kappa$ is the integer part of $\delta / \varepsilon$, i.e., $\kappa=$ $\lfloor\delta / \varepsilon\rfloor$. Define a test function $\varphi \equiv \theta^{\varepsilon}+\left(\widehat{W}^{\varepsilon}-W_{\ell}\right)\left(1-\rho^{\varepsilon}\right)$, where the cut-off function $\rho^{\varepsilon} \in C_{0}^{\infty}\left(I_{\delta}\right),\left|\nabla \rho^{\varepsilon}\right| \leq C / \varepsilon$, and

$$
\rho^{\varepsilon}(\boldsymbol{x})= \begin{cases}1, & \text { if } \operatorname{dist}\left(\boldsymbol{x}, \partial I_{\delta}\right) \geq 2 \varepsilon, \\ 0, & \text { if } \operatorname{dist}\left(\boldsymbol{x}, \partial I_{\delta}\right) \leq \varepsilon .\end{cases}
$$

Multiplying both sides of $(3.12)_{1}$ by $\varphi$, integrating by parts and using the fact that $\varphi \in H_{0}^{1}\left(I_{\delta}\right)$, we have

$$
\int_{I_{\delta}\left(\boldsymbol{x}_{\ell}\right)} \nabla \theta^{\varepsilon} \cdot a_{\ell}^{\varepsilon} \nabla \theta^{\varepsilon} \mathrm{d} \boldsymbol{x}=\int_{I_{\delta}\left(\boldsymbol{x}_{\ell}\right)} \nabla\left[\left(W_{\ell}-\widehat{W}^{\varepsilon}\right)\left(1-\rho^{\varepsilon}\right)\right] \cdot a_{\ell}^{\varepsilon} \nabla \theta^{\varepsilon} \mathrm{d} \boldsymbol{x} .
$$

By the ellipticity of $a_{\ell}^{\varepsilon}$, we obtain

$$
\left\|\nabla \theta^{\varepsilon}\right\|_{L^{2}\left(I_{\delta}\right)} \leq \frac{\Lambda}{\lambda}\left\|\nabla\left[\left(\widehat{W}^{\varepsilon}-W_{\ell}\right)\left(1-\rho^{\varepsilon}\right)\right]\right\|_{L^{2}\left(I_{\delta}\right)} .
$$

Using (3.5), we obtain

$$
\begin{aligned}
\left\|\left(\widehat{W}^{\varepsilon}-W_{\ell}\right) \nabla\left(1-\rho^{\varepsilon}\right)\right\|_{L^{2}\left(I_{\delta}\right)} & \leq C\|\chi\|_{L^{\infty}(Y)}\left\|\nabla W_{\ell}\right\|_{L^{2}\left(I_{\delta} \backslash I_{\kappa \varepsilon}\right)} \\
& \leq C\left(\frac{\varepsilon}{\delta}\right)^{1 / 2}\left\|\nabla W_{\ell}\right\|_{L^{2}\left(I_{\delta}\right)},
\end{aligned}
$$

and

$$
\begin{aligned}
\left\|\left(1-\rho^{\varepsilon}\right) \nabla\left(\widehat{W}^{\varepsilon}-W_{\ell}\right)\right\|_{L^{2}\left(I_{\delta}\right)} & \leq C\left\|\nabla_{\boldsymbol{y}} \chi\right\|_{L^{\infty}(Y)}\left\|\nabla W_{\ell}\right\|_{L^{2}\left(I_{\delta} \backslash I_{\kappa \varepsilon}\right)} \\
& \leq C\left(\frac{\varepsilon}{\delta}\right)^{1 / 2}\left\|\nabla W_{\ell}\right\|_{L^{2}\left(I_{\delta}\right)} .
\end{aligned}
$$


Combining the above two inequalities, we obtain

$$
\left\|\nabla\left[\left(\widehat{W}^{\varepsilon}-W_{\ell}\right)\left(1-\rho^{\varepsilon}\right)\right]\right\|_{L^{2}\left(I_{\delta}\right)} \leq C\left(\frac{\varepsilon}{\delta}\right)^{1 / 2}\left\|\nabla W_{\ell}\right\|_{L^{2}\left(I_{\delta}\right)},
$$

which gives (3.13).

The next lemma concerns the estimate of $\left\|(\mathcal{A}-\widehat{\mathcal{A}})\left(\boldsymbol{x}_{\ell}\right)\right\|$ at any point $\boldsymbol{x}_{\ell}$.

Lemma 3.7. There exists $C$ such that for any $\boldsymbol{x}_{\ell} \in K$,

$$
\left\|(\mathcal{A}-\widehat{\mathcal{A}})\left(\boldsymbol{x}_{\ell}\right)\right\| \leq C \frac{\varepsilon}{\delta}
$$

Proof. Integrating by parts with (3.11), we obtain

$$
f_{I_{\kappa \varepsilon}} \nabla\left(\widehat{V}^{\varepsilon}-V_{\ell}\right) \cdot a_{\ell}^{\varepsilon} \nabla \widehat{W}^{\varepsilon} \mathrm{d} \boldsymbol{x}=0
$$

Using the expressions of $\widehat{W}^{\varepsilon}$ and $\mathcal{A}$, we obtain

$$
f_{I_{\kappa \varepsilon}} \nabla V_{\ell} \cdot a_{\ell}^{\varepsilon} \nabla \widehat{W}^{\varepsilon} \mathrm{d} \boldsymbol{x}=\nabla V_{\ell} \cdot \mathcal{A}\left(\boldsymbol{x}_{\ell}\right) \nabla W_{\ell} .
$$

It follows from the above two equations that

$$
f_{I_{\kappa \varepsilon}} \nabla \widehat{V}^{\varepsilon} \cdot a_{\ell}^{\varepsilon} \nabla \widehat{W}^{\varepsilon} \mathrm{d} \boldsymbol{x}=\nabla V_{\ell} \cdot \mathcal{A}\left(\boldsymbol{x}_{\ell}\right) \nabla W_{\ell}
$$

By the definition of $\widehat{\mathcal{A}}$, we have

$$
\begin{aligned}
& (\nabla V \cdot(\mathcal{A}-\widehat{\mathcal{A}}) \nabla W)\left(\boldsymbol{x}_{\ell}\right) \\
& =\left(1-\frac{\left|I_{\kappa \varepsilon}\right|}{\left|I_{\delta}\right|}\right) f_{I_{\kappa \varepsilon}} \nabla \widehat{V}^{\varepsilon} \cdot a_{\ell}^{\varepsilon} \nabla \widehat{W}^{\varepsilon} \mathrm{d} \boldsymbol{x}-\frac{1}{\left|I_{\delta}\right|} \int_{I_{\delta} \backslash I_{\kappa \varepsilon}} \nabla \widehat{V}^{\varepsilon} \cdot a_{\ell}^{\varepsilon} \nabla \widehat{W}^{\varepsilon} \mathrm{d} \boldsymbol{x} \\
& =\left(1-\frac{\left|I_{\kappa \varepsilon}\right|}{\left|I_{\delta}\right|}\right) \nabla V_{\ell} \cdot \mathcal{A}\left(\boldsymbol{x}_{\ell}\right) \nabla W_{\ell}-\frac{1}{\left|I_{\delta}\right|} \int_{I_{\delta} \backslash I_{\kappa \varepsilon}} \nabla \widehat{V}^{\varepsilon} \cdot a_{\ell}^{\varepsilon} \nabla \widehat{W}^{\varepsilon} \mathrm{d} \boldsymbol{x} .
\end{aligned}
$$

In view of (3.5) and the definitions of $\widehat{V}^{\varepsilon}$ and $\widehat{W}^{\varepsilon}$, we have

$$
\left\|\nabla \widehat{V}^{\varepsilon}\right\|_{L^{2}\left(I_{\delta} \backslash I_{\kappa \varepsilon}\right)} \leq C\left\|\nabla V_{\ell}\right\|_{L^{2}\left(I_{\delta} \backslash I_{\kappa \varepsilon}\right)} \leq C\left(\frac{\varepsilon}{\delta}\right)^{1 / 2}\left\|\nabla V_{\ell}\right\|_{L^{2}\left(I_{\delta}\right)},
$$

and similarly,

$$
\left\|\nabla \widehat{W}^{\varepsilon}\right\|_{L^{2}\left(I_{\delta} \backslash I_{\kappa \varepsilon}\right)} \leq C\left(\frac{\varepsilon}{\delta}\right)^{1 / 2}\left\|\nabla W_{\ell}\right\|_{L^{2}\left(I_{\delta}\right)}
$$

Using the above estimates and the ellipticity of $\mathcal{A}$, we obtain

$$
\left|(\nabla V \cdot(\mathcal{A}-\widehat{\mathcal{A}}) \nabla W)\left(\boldsymbol{x}_{\ell}\right)\right| \leq C \frac{\varepsilon}{\delta}\left|\nabla V_{\ell}\right|\left|\nabla W_{\ell}\right|,
$$

which in turn implies (3.15). 
Now we bound $\left\|\left(\widetilde{\mathcal{A}}_{H}-\widehat{\mathcal{A}}\right)\left(\boldsymbol{x}_{\ell}\right)\right\|$ for any $\boldsymbol{x}_{\ell} \in K$. The proof is based on the identity (3.10).

Lemma 3.8. There exists $C$ such that

$$
\left\|\left(\widetilde{\mathcal{A}}_{H}-\widehat{\mathcal{A}}\right)\left(\boldsymbol{x}_{\ell}\right)\right\| \leq C \frac{\varepsilon}{\delta} .
$$

Proof. Define a test function $\varphi \equiv \widehat{v}^{\varepsilon}-\widehat{V}^{\varepsilon}+\left(\widehat{V}^{\varepsilon}-V_{\ell}\right)\left(1-\rho^{\varepsilon}\right)$, where $\rho^{\varepsilon}$ is defined in Lemma 3.6. Multiplying both sides of (3.11) by $\varphi$, integrating by parts and using the fact that $\varphi \in H_{0}^{1}\left(I_{\delta}\right)$, we obtain

$$
f_{I_{\delta}\left(\boldsymbol{x}_{\ell}\right)} \nabla\left(\widehat{v}^{\varepsilon}-\widehat{V}^{\varepsilon}\right) \cdot a_{\ell}^{\varepsilon} \nabla \widehat{W}^{\varepsilon} \mathrm{d} \boldsymbol{x}=f_{I_{\delta}\left(\boldsymbol{x}_{\ell}\right)} \nabla\left[\left(V_{\ell}-\widehat{V}^{\varepsilon}\right)\left(1-\rho^{\varepsilon}\right)\right] \cdot a_{\ell}^{\varepsilon} \nabla \widehat{W}^{\varepsilon} \mathrm{d} \boldsymbol{x} .
$$

Using (3.17) and (3.14), we obtain

$$
\left|f_{I_{\delta}\left(\boldsymbol{x}_{\ell}\right)} \nabla\left(\widehat{v}^{\varepsilon}-\widehat{V}^{\varepsilon}\right) \cdot a_{\ell}^{\varepsilon} \nabla \widehat{W}^{\varepsilon} \mathrm{d} \boldsymbol{x}\right| \leq C \frac{\varepsilon}{\delta}\left|\nabla V_{\ell}\right|\left|\nabla W_{\ell}\right| .
$$

Using the fact that $\widetilde{v}^{\varepsilon}=\widehat{v}^{\varepsilon}$ on $\partial I_{\delta}\left(\boldsymbol{x}_{\ell}\right)$, integrating by parts with $(3.12)_{1}$ we obtain

$$
\begin{aligned}
f_{I_{\delta}\left(\boldsymbol{x}_{\ell}\right)} \nabla \widehat{v}^{\varepsilon} \cdot a_{\ell}^{\varepsilon} \nabla\left(\widehat{w}^{\varepsilon}-\widehat{W}^{\varepsilon}\right) \mathrm{d} \boldsymbol{x} & =f_{I_{\delta}\left(\boldsymbol{x}_{\ell}\right)} \nabla \widetilde{v}^{\varepsilon} \cdot a_{\ell}^{\varepsilon} \nabla\left(\widehat{w}^{\varepsilon}-\widehat{W}^{\varepsilon}\right) \mathrm{d} \boldsymbol{x} \\
& =f_{I_{\delta}\left(\boldsymbol{x}_{\ell}\right)} \nabla\left(\widehat{w}^{\varepsilon}-\widehat{W}^{\varepsilon}\right) \cdot\left(a_{\ell}^{\varepsilon}\right)^{t} \nabla \widetilde{v}^{\varepsilon} \mathrm{d} \boldsymbol{x} \\
& =f_{I_{\delta}\left(\boldsymbol{x}_{\ell}\right)} \nabla\left[\left(W_{\ell}-\widehat{W}^{\varepsilon}\right)\left(1-\rho^{\varepsilon}\right)\right] \cdot\left(a_{\ell}^{\varepsilon}\right)^{t} \nabla \widetilde{v}^{\varepsilon} \mathrm{d} \boldsymbol{x} .
\end{aligned}
$$

Let $\widetilde{V}^{\varepsilon}=V_{\ell}+\varepsilon\left(\widetilde{\boldsymbol{\chi}}_{\ell} \cdot \nabla\right) V_{\ell}$, where $\widetilde{\boldsymbol{\chi}}_{\ell}$ is the solution of (3.4) with $a(\boldsymbol{x}, \boldsymbol{y})$ replaced by $\left(a\left(\boldsymbol{x}_{\ell}, \boldsymbol{y}\right)\right)^{t}$. By $(3.13)$ and (3.16), we obtain

$$
\left\|\nabla \widetilde{v}^{\varepsilon}\right\|_{L^{2}\left(I_{\delta} \backslash I_{\kappa \varepsilon}\right)} \leq\left\|\nabla\left(\widetilde{v}^{\varepsilon}-\widetilde{V}^{\varepsilon}\right)\right\|_{L^{2}\left(I_{\delta}\right)}+\left\|\nabla \widetilde{V}^{\varepsilon}\right\|_{L^{2}\left(I_{\delta} \backslash I_{\kappa \varepsilon}\right)} \leq C\left(\frac{\varepsilon}{\delta}\right)^{1 / 2}\left\|\nabla V_{\ell}\right\|_{L^{2}\left(I_{\delta}\right)},
$$

which together with (3.14) gives

$$
\begin{aligned}
\left|f_{I_{\delta}\left(\boldsymbol{x}_{\ell}\right)} \nabla \widehat{v}^{\varepsilon} \cdot a_{\ell}^{\varepsilon} \nabla\left(\widehat{w}^{\varepsilon}-\widehat{W}^{\varepsilon}\right) \mathrm{d} \boldsymbol{x}\right| & \leq \frac{\Lambda}{\left|I_{\delta}\right|}\left\|\nabla\left[\left(W_{\ell}-\widehat{W}^{\varepsilon}\right)\left(1-\rho^{\varepsilon}\right)\right]\right\|_{L^{2}\left(I_{\delta}\right)}\left\|\nabla \widetilde{v}^{\varepsilon}\right\|_{L^{2}\left(I_{\delta} \backslash I_{\kappa \varepsilon}\right)} \\
& \leq C \frac{\varepsilon}{\delta}\left|\nabla V_{\ell}\right|\left|\nabla W_{\ell}\right| .
\end{aligned}
$$

Combining the above estimate with (3.19), we get (3.18).

Proof of Lemma 3.5. The estimate (3.9) follows from (3.15) and (3.18).

It remains to bound $\left\|\left(\mathcal{A}_{H}-\widetilde{\mathcal{A}}_{H}\right)\left(\boldsymbol{x}_{\ell}\right)\right\|$, which concerns the accuracy of the microscopic solver. The main ingredient is the following regularity result:

Lemma 3.9. Let $\widehat{w}^{\varepsilon}-W_{\ell} \in H_{0}^{1}\left(I_{\delta}\right)$ satisfy (3.7). Then there exists $C$ that is independent of $\varepsilon$ and $\delta$ such that

$$
\left\|\nabla \widehat{w}^{\varepsilon}\right\|_{H^{m}\left(I_{\delta}\right)} \leq C \varepsilon^{-m}\left\|\nabla W_{\ell}\right\|_{L^{2}\left(I_{\delta}\right)}, \quad m=0,1 .
$$


The estimate (3.20) is quite standard except that the constant $C$ is independent of the cell size $\delta$. In the appendix we provide a proof that is based on the second fundamental inequality for the elliptic operator [19].

Now we are ready to prove Theorem 3.3.

Proof of Theorem 3.3. The proof is based on the following identity:

$$
\begin{aligned}
& \left(\nabla V \cdot\left(\mathcal{A}_{H}-\widetilde{\mathcal{A}}_{H}\right) \nabla W\right)\left(\boldsymbol{x}_{\ell}\right) \\
& =f_{I_{\delta}\left(\boldsymbol{x}_{\ell}\right)}\left[\nabla v_{h}^{\varepsilon} \cdot\left(a^{\varepsilon}-a_{\ell}^{\varepsilon}\right) \nabla w_{h}^{\varepsilon}+\nabla v_{h}^{\varepsilon} \cdot a_{\ell}^{\varepsilon} \nabla\left(w_{h}^{\varepsilon}-\widehat{w}_{h}^{\varepsilon}\right)\right] \mathrm{d} \boldsymbol{x} \\
& \quad+f_{I_{\delta}\left(\boldsymbol{x}_{\ell}\right)}\left[\nabla v_{h}^{\varepsilon} \cdot a_{\ell}^{\varepsilon} \nabla \widehat{w}_{h}^{\varepsilon}-\nabla \widehat{v}^{\varepsilon} \cdot a_{\ell}^{\varepsilon} \nabla \widehat{w}^{\varepsilon}\right] \mathrm{d} \boldsymbol{x} .
\end{aligned}
$$

It follows from the definitions of $w_{h}^{\varepsilon}$ and $\widehat{w}_{h}^{\varepsilon}$ that

$$
\begin{aligned}
\lambda\left\|\nabla\left(w_{h}^{\varepsilon}-\widehat{w}_{h}^{\varepsilon}\right)\right\|_{L^{2}\left(I_{\delta}\right)}^{2} & \leq \int_{I_{\delta}\left(\boldsymbol{x}_{\ell}\right)} \nabla\left(w_{h}^{\varepsilon}-\widehat{w}_{h}^{\varepsilon}\right) \cdot a_{\ell}^{\varepsilon} \nabla\left(w_{h}^{\varepsilon}-\widehat{w}_{h}^{\varepsilon}\right) \mathrm{d} \boldsymbol{x} \\
& =\int_{I_{\delta}\left(\boldsymbol{x}_{\ell}\right)} \nabla\left(w_{h}^{\varepsilon}-\widehat{w}_{h}^{\varepsilon}\right) \cdot a_{\ell}^{\varepsilon} \nabla w_{h}^{\varepsilon} \mathrm{d} \boldsymbol{x} \\
& =\int_{I_{\delta}\left(\boldsymbol{x}_{\ell}\right)} \nabla\left(w_{h}^{\varepsilon}-\widehat{w}_{h}^{\varepsilon}\right) \cdot\left(a_{\ell}^{\varepsilon}-a^{\varepsilon}\right) \nabla w_{h}^{\varepsilon} \mathrm{d} \boldsymbol{x} .
\end{aligned}
$$

If $a(\boldsymbol{x}, \boldsymbol{y}) \in C^{0,1}\left(D ; L^{\infty}(Y)\right)$, then

$$
\left\|\nabla\left(w_{h}^{\varepsilon}-\widehat{w}_{h}^{\varepsilon}\right)\right\|_{L^{2}\left(I_{\delta}\right)} \leq C \delta\left\|\nabla w_{h}^{\varepsilon}\right\|_{L^{2}\left(I_{\delta}\right)} \leq C \delta\left\|\nabla W_{\ell}\right\|_{L^{2}\left(I_{\delta}\right)},
$$

where we have used the estimate (3.3) for $w_{h}^{\varepsilon}$. Using the above inequality, we bound the first term in (3.21) as

$$
\left|f_{I_{\delta}\left(\boldsymbol{x}_{\ell}\right)}\left[\nabla v_{h}^{\varepsilon} \cdot\left(a^{\varepsilon}-a_{\ell}^{\varepsilon}\right) \nabla w_{h}^{\varepsilon}+\nabla v_{h}^{\varepsilon} \cdot a_{\ell}^{\varepsilon} \nabla\left(w_{h}^{\varepsilon}-\widehat{w}_{h}^{\varepsilon}\right)\right] \mathrm{d} \boldsymbol{x}\right| \leq C \delta\left|\nabla V_{\ell}\right|\left|\nabla W_{\ell}\right| .
$$

Taking $z=\widetilde{v}_{h}^{\varepsilon}-\widehat{v}^{\varepsilon} \in H_{0}^{1}\left(I_{\delta}\right)$ in (3.7), we obtain

$$
\int_{I_{\delta}\left(\boldsymbol{x}_{\ell}\right)} \nabla \widehat{v}^{\varepsilon} \cdot a_{\ell}^{\varepsilon} \nabla \widehat{w}^{\varepsilon} \mathrm{d} \boldsymbol{x}=\int_{I_{\delta}\left(\boldsymbol{x}_{\ell}\right)} \nabla \widehat{v}_{h}^{\varepsilon} \cdot a_{\ell}^{\varepsilon} \nabla \widehat{w}^{\varepsilon} \mathrm{d} \boldsymbol{x} .
$$

Similarly,

$$
\int_{I_{\delta}\left(\boldsymbol{x}_{\ell}\right)} \nabla\left(\widehat{w}_{h}^{\varepsilon}-\widehat{w}^{\varepsilon}\right) \cdot\left(a_{\ell}^{\varepsilon}\right)^{t} \nabla \widetilde{v}^{\varepsilon} \mathrm{d} \boldsymbol{x}=0
$$

Setting $z=\widetilde{v}_{h}^{\varepsilon}-v_{h}^{\varepsilon} \in X_{h}$ in the variational formulation for $\widehat{w}_{h}^{\varepsilon}$, which is defined in the same manner as (2.3) with $a^{\varepsilon}$ replaced by $a_{\ell}^{\varepsilon}$, we have

$$
\int_{I_{\delta}\left(\boldsymbol{x}_{\ell}\right)} \nabla v_{h}^{\varepsilon} \cdot a_{\ell}^{\varepsilon} \nabla \widehat{w}_{h}^{\varepsilon} \mathrm{d} \boldsymbol{x}=\int_{I_{\delta}\left(\boldsymbol{x}_{\ell}\right)} \nabla \widetilde{v}_{h}^{\varepsilon} \cdot a_{\ell}^{\varepsilon} \nabla \widehat{w}_{h}^{\varepsilon} \mathrm{d} \boldsymbol{x} .
$$


It follows from the above three equations that

$$
\begin{aligned}
f_{I_{\delta}\left(\boldsymbol{x}_{\ell}\right)}\left[\nabla v_{h}^{\varepsilon} \cdot a_{\ell}^{\varepsilon} \nabla \widehat{w}_{h}^{\varepsilon}\right. & \left.-\nabla \widehat{v}^{\varepsilon} \cdot a_{\ell}^{\varepsilon} \nabla \widehat{w}^{\varepsilon}\right] \mathrm{d} \boldsymbol{x}=f_{I_{\delta}\left(\boldsymbol{x}_{\ell}\right)} \nabla \widetilde{v}_{h}^{\varepsilon} \cdot a_{\ell}^{\varepsilon} \nabla\left(\widehat{w}_{h}^{\varepsilon}-\widehat{w}^{\varepsilon}\right) \mathrm{d} \boldsymbol{x} \\
& =f_{I_{\delta}\left(\boldsymbol{x}_{\ell}\right)} \nabla\left(\widehat{w}_{h}^{\varepsilon}-\widehat{w}^{\varepsilon}\right) \cdot\left(a_{\ell}^{\varepsilon}\right)^{t} \nabla \widetilde{v}_{h}^{\varepsilon} \mathrm{d} \boldsymbol{x} \\
& =f_{I_{\delta}\left(\boldsymbol{x}_{\ell}\right)} \nabla\left(\widehat{w}_{h}^{\varepsilon}-\widehat{w}^{\varepsilon}\right) \cdot\left(a_{\ell}^{\varepsilon}\right)^{t} \nabla\left(\widetilde{v}_{h}^{\varepsilon}-\widetilde{v}^{\varepsilon}\right) \mathrm{d} \boldsymbol{x} .
\end{aligned}
$$

Using the standard error estimate for linear finite elements and the regularity estimate (3.20), we obtain

$$
\left\|\nabla\left(\widehat{w}^{\varepsilon}-\widehat{w}_{h}^{\varepsilon}\right)\right\|_{L^{2}\left(I_{\delta}\right)} \leq C \frac{h}{\varepsilon}\left\|\nabla W_{\ell}\right\|_{L^{2}\left(I_{\delta}\right)}, \quad\left\|\nabla\left(\widetilde{v}^{\varepsilon}-\widetilde{v}_{h}^{\varepsilon}\right)\right\|_{L^{2}\left(I_{\delta}\right)} \leq C \frac{h}{\varepsilon}\left\|\nabla V_{\ell}\right\|_{L^{2}\left(I_{\delta}\right)},
$$

which leads to

$$
\left|f_{I_{\delta}\left(\boldsymbol{x}_{\ell}\right)}\left[\nabla v_{h}^{\varepsilon} \cdot a_{\ell}^{\varepsilon} \nabla \widehat{w}_{h}^{\varepsilon}-\nabla \widehat{v}^{\varepsilon} \cdot a_{\ell}^{\varepsilon} \nabla \widehat{w}^{\varepsilon}\right] \mathrm{d} \boldsymbol{x}\right| \leq C \frac{h^{2}}{\varepsilon^{2}}\left|\nabla V_{\ell}\right|\left|\nabla W_{\ell}\right| .
$$

This together with (3.22) implies

$$
\left\|\left(\widetilde{\mathcal{A}}_{H}-\mathcal{A}_{H}\right)\left(\boldsymbol{x}_{\ell}\right)\right\| \leq C\left(\delta+\frac{h^{2}}{\varepsilon^{2}}\right) .
$$

Combining the above estimate and (3.9), we obtain (3.6).

When the cell problem (2.3) is supplemented with the periodic boundary condition, the microscopic finite element space $X_{h}$ is defined as

$$
X_{h} \equiv\left\{v \in H_{\#}^{1}\left(I_{\delta}\left(\boldsymbol{x}_{\ell}\right)\right)|v|_{K} \in P_{k^{\prime}}(K), K \in \mathcal{T}_{h}, \text { and } \int_{I_{\delta}\left(\boldsymbol{x}_{\ell}\right)} v \mathrm{~d} \boldsymbol{x}=0\right\},
$$

where $H_{\#}^{1}\left(I_{\delta}\left(\boldsymbol{x}_{\ell}\right)\right)$ is the closure of $C_{\#}^{\infty}\left(I_{\delta}\left(\boldsymbol{x}_{\ell}\right)\right)$ for the $H^{1}$ norm, and $C_{\#}^{\infty}\left(I_{\delta}\left(\boldsymbol{x}_{\ell}\right)\right)$ is the subset of $C^{\infty}\left(I_{\delta}\left(\boldsymbol{x}_{\ell}\right)\right)$ of $I_{\delta}\left(\boldsymbol{x}_{\ell}\right)$-periodic functions [12].

Corollary 3.10. Let $\delta=\mathbb{N} \varepsilon$ with $\mathbb{N}$ an integer, and solve (2.3) with a periodic boundary condition. If Assumption (3.5) holds true for $s=k^{\prime}+1$, then

$$
e(\mathrm{HMM}) \leq C\left(\delta+\left(\frac{h}{\varepsilon}\right)^{2 k^{\prime}}\right)
$$

Proof. It is easy to check that

$$
\widehat{w}^{\varepsilon}=W_{\ell}+\varepsilon\left(\chi_{\ell} \cdot \nabla\right) W_{\ell} \quad \text { and } \quad \widehat{w}_{h}^{\varepsilon}=W_{\ell}+\varepsilon\left(\chi_{\ell}^{h} \cdot \nabla\right) W_{\ell},
$$

where $\chi_{\ell}^{h}$ is the finite element approximation of $\chi_{\ell}$ associated with Problem (3.4) and the finite element space $X_{h}$ defined in (3.24). It follows from the standard finite element error estimate and (3.5) that

$$
\begin{aligned}
\left\|\nabla\left(\widehat{w}^{\varepsilon}-\widehat{w}_{h}^{\varepsilon}\right)\right\|_{L^{2}\left(I_{\delta}\right)} & =\varepsilon\left\|\nabla\left(\chi_{\ell}-\chi_{\ell}^{h}\right)\right\|_{L^{2}\left(I_{\delta}\right)}\left|\nabla W_{\ell}\right| \\
& \leq C \varepsilon h^{k^{\prime}}\left\|\nabla^{k^{\prime}+1} \chi_{\ell}\right\|_{L^{2}\left(I_{\delta}\right)}\left|\nabla W_{\ell}\right| \\
& \leq C \varepsilon h^{k^{\prime}}\left\|\nabla^{k^{\prime}+1} \chi_{\ell}\right\|_{L^{\infty}(Y)}\left\|\nabla W_{\ell}\right\|_{L^{2}\left(I_{\delta}\right)} \\
& \leq C\left(\frac{h}{\varepsilon}\right)^{k^{\prime}}\left\|\nabla W_{\ell}\right\|_{L^{2}\left(I_{\delta}\right)} .
\end{aligned}
$$


Since $\widehat{w}^{\varepsilon}=\widehat{W}^{\varepsilon}$ and $\delta=\mathbb{N} \varepsilon$, we have

$$
(\nabla V \cdot \mathcal{A} \nabla W)\left(\boldsymbol{x}_{\ell}\right)=f_{I_{\delta}\left(\boldsymbol{x}_{\ell}\right)} \nabla \widehat{v}^{\varepsilon} \cdot a_{\ell}^{\varepsilon} \nabla \widehat{w}^{\varepsilon} \mathrm{d} \boldsymbol{x}
$$

Replacing $\widetilde{\mathcal{A}}_{H}$ by $\mathcal{A}$ in (3.21), proceeding along the same lines that lead to (3.23), and using (3.26), we obtain (3.25).

In [1, 2], Assyr proved (3.25) for the case when $a^{\varepsilon}=\left(a^{\varepsilon}\right)^{t}, \delta=\varepsilon, a_{\ell}^{\varepsilon}$ in place of $a^{\varepsilon}$ and the periodic boundary condition is employed in the cell problem (2.3), i.e., he proved

$$
e(\mathrm{HMM}) \leq C(h / \varepsilon)^{2 k^{\prime}}
$$

REMARK 3.11. We have proved Theorem 3.3 under the assumption (3.5), which is quite common in the analysis of HMM $[16,23,1]$. In case of $s=1$, it has been proved to be true for certain interesting cases (see [20]). However, this assumption is not true for real composite materials [7], even for $s=1$. Actually, this assumption has been removed recently by the authors in [14].

\section{Numerical examples}

Let the domain D be a unit square $(0,1)^{2}$. We firstly triangulate D into $N \times N$ squares and then divide each square into two sub-triangles along its diagonal with positive slope. Similarly, we triangulate each microcell into $\mathrm{M} \times \mathrm{M}$ squares and then divide each square into two sub-triangles along its diagonal with positive slope; see figure 4.1. We solve (1.1) with HMM-FEM (2.1), and the resulting linear systems are solved by a parallel sparse direct solver $M U M P S$ [24]. Total CPU time is recorded on the SGI Origin3800 as a measure of the computational cost. We emphasize that the CPU time instead of the total number of the degrees of freedom is used as the a measure of cost since the dominant cost in HMM-FEM is the cost on numerically solving the cell problem. Other costs are negligible; see [22, 1].

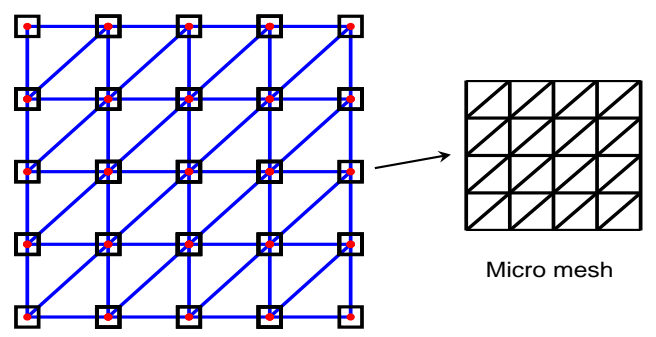

Macro mesh

FIgURE 4.1. Schematic showcase of triangulation over $D$ and microcell that is located at the vertex. The total number of microcells for the schemes (2.7), (2.8), (2.9), and (2.10) are $25,32,56$, and 96 , respectively.

It is more transparent to reshape the error estimates in Theorems 3.2 and 3.3 in 
terms of $N$ and $M$. For $k=1,2$,

$$
\begin{aligned}
\left\|U_{0}-U_{H}\right\|_{H^{1}(D)} & \leq C\left(N^{-k}+e(\mathrm{HMM})\right), \\
\left\|U_{0}-U_{H}\right\|_{L^{2}(D)} & \leq C\left(N^{-(k+1)}+e(\mathrm{HMM})\right), \\
e(\mathrm{HMM}) & \leq C\left(\delta+\frac{\varepsilon}{\delta}+M^{\prime-2}\right),
\end{aligned}
$$

where $M^{\prime} \equiv M \varepsilon / \delta$, which signals the resolution per wave length and the term $\delta+\varepsilon / \delta$ in the right-hand side of $e(\mathrm{HMM})$ represents the error caused by estimating the effective matrix. The term $\varepsilon / \delta$ is dubbed as the resonance error by the authors in [18].

As a starting point, we investigate the accuracy of the microscopic solver and order of the resonance error through the following example.

EXAMPLe 4.1. Consider the following matrix $a^{\varepsilon}$ that is non-symmetric and each entry is a strictly periodic function.

$$
a(\boldsymbol{x} / \varepsilon)=\left(\begin{array}{ll}
5+\frac{3}{2} \cos \frac{2 \pi x_{1}}{\varepsilon} & 2+\sin \frac{2 \pi x_{1}}{\varepsilon} \\
3+2 \cos \frac{2 \pi x_{1}}{\varepsilon} & 6+2 \sin \frac{2 \pi x_{1}}{\varepsilon}
\end{array}\right)
$$

with $\varepsilon=1 / 100$.

Using [12, Theorem 5.10], a direct calculation gives the explicit formula of the effective matrix as

$$
\mathcal{A}=\left(\begin{array}{cc}
\frac{\sqrt{91}}{2} & 2 \\
\frac{2 \sqrt{91}}{3}-\frac{11}{3} & 6
\end{array}\right) .
$$

In practice, we take $W_{\ell}$ as the canonical basis $x_{i}=\boldsymbol{e}_{i} \cdot \boldsymbol{x}$ in (2.3) and each entry of $\mathcal{A}_{H}$ is evaluated by (2.4).

Accuracy of microscopic solver. Choose $\delta=\varepsilon$ and solve the cell problem (2.3) with periodic boundary conditions: find $\phi_{i}^{\varepsilon}-x_{i} \in X_{h}$ such that

$$
\int_{I_{\delta}} \nabla z \cdot a(\boldsymbol{x} / \varepsilon) \nabla \phi_{i}^{\varepsilon} \mathrm{d} \boldsymbol{x}=0 \quad \text { for all } \quad z \in X_{h} .
$$

The effective matrix $\mathcal{A}_{H}$ is given by

$$
\left(\mathcal{A}_{H}\right)_{i j}=\boldsymbol{e}_{i} \cdot \mathcal{A}_{H} \boldsymbol{e}_{j}=\nabla x_{i} \cdot \mathcal{A}_{H} \nabla x_{j}=f_{I_{\delta}} \nabla \phi_{i}^{\varepsilon} \cdot a(\boldsymbol{x} / \varepsilon) \nabla \phi_{j}^{\varepsilon} \mathrm{d} \boldsymbol{x} .
$$

The estimate (3.25) changes to

$$
e(\mathrm{HMM}) \leq C M^{-2 k^{\prime}} .
$$

Adopting different micro mesh sizes, we obtain the log-log plot of $e(\mathrm{HMM})$ in terms of $M$ for $k^{\prime}=1,2$. figure 4.2 confirms the sharpness of the above estimate.

Order of the resonance error. Choose $\delta=L \varepsilon$, where $L$ is not necessarily an integer. The cell problems are solved over a $1024 \times 1024$ mesh by linear finite elements with a 

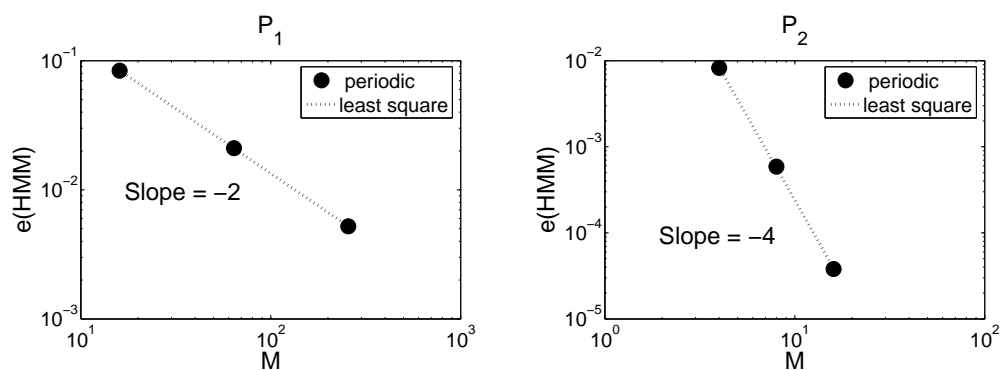

Figure 4.2. Accuracy of the microscopic solvers. Left: $P_{1}$; Right: $P_{2}$.

Dirichlet boundary condition, and $\mathcal{A}_{H}$ is computed by (4.5). The estimate (4.3) now changes to

$$
e(\mathrm{HMM}) \leq C\left(L^{-1}+L^{2} M^{-2}\right), \quad M=1024 .
$$

Obviously, the dominant term in the right hand side of $e(\mathrm{HMM})$ is $\mathcal{O}\left(L^{-1}\right)$. Adopting different cell sizes, we obtain the $\log -\log$ plot of $e(\mathrm{HMM})$ in terms of $L$. By figure 4.3, we can see the clear first order convergence that is conforming with the above estimate.

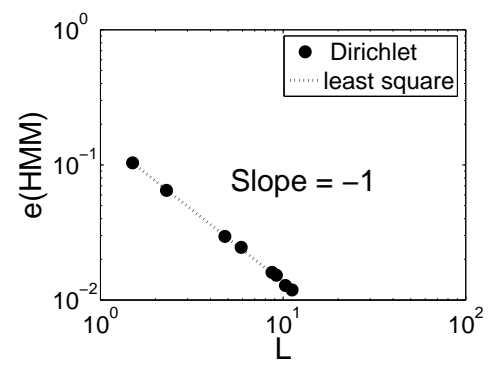

Figure 4.3. Order of the resonance error.

Next we study the effect of different numerical integration schemes and different macroscopic solvers. Solve (1.1) with

EXAMPLE 4.2.

$$
\left\{\begin{aligned}
& a(\boldsymbol{x}, \boldsymbol{x} / \varepsilon)=\frac{\left(R_{1}+R_{2} \sin \left(2 \pi x_{1}\right)\right)\left(R_{1}+R_{2} \cos \left(2 \pi x_{2}\right)\right)}{\left(R_{1}+R_{2} \sin \frac{2 \pi x_{1}}{\varepsilon}\right)\left(R_{1}+R_{2} \sin \frac{2 \pi x_{2}}{\varepsilon}\right)} I, \\
& f=1, \quad u=0 \quad \text { on } \partial D
\end{aligned}\right.
$$

where $I$ is the identity matrix of order 2 and $\varepsilon=1 / 100$. This coefficient is taken from [18]. A direct calculation gives the explicit formula of the effective matrix as

$$
\mathcal{A}\left(x_{1}, x_{2}\right)=\frac{\left(R_{1}+R_{2} \sin \left(2 \pi x_{1}\right)\right)\left(R_{1}+R_{2} \cos \left(2 \pi x_{2}\right)\right)}{R_{1} \sqrt{R_{1}^{2}-R_{2}^{2}}} I .
$$


We take $R_{1}=2.5$ and $R_{2}=1.5$ in the simulation. We compute $U_{0}$ by solving (1.2) with the above expression of $\mathcal{A}$ over a $256 \times 256$ mesh with linear finite elements. To obtain $U_{H}$, we let $\delta=\varepsilon$ and solve the cell problems (2.3) with periodic boundary condition as in (4.4). The coefficient $a(\boldsymbol{x}, \boldsymbol{x} / \varepsilon)$ in $(2.3)$ is replaced by $a\left(\boldsymbol{x}_{\ell}, \boldsymbol{x} / \varepsilon\right)$. For convenience, we will use the notation " $P_{k}$-type of numerical integration scheme" to describe the solvers. Using (4.1), (4.2), and Corollary 3.10, we have, for $k=1,2$,

$$
\begin{aligned}
&\left\|U_{0}-U_{H}\right\|_{H^{1}(D)} \leq C\left(N^{-k}+M^{-2}\right), \\
&\left\|U_{0}-U_{H}\right\|_{L^{2}(D)} \leq C\left(N^{-(k+1)}+M^{-2}\right) .
\end{aligned}
$$

The above estimate suggests the following refinement strategy on the microcell:

$$
M= \begin{cases}N^{k / 2} & H^{1} \text { error } \\ N^{(k+1) / 2} & L^{2} \text { error. }\end{cases}
$$

We note that the same strategy has been proposed in [1] when $k=1$. We report the results in Tables 4.1-4.4, which are based on the above choice. For clarity of the comparison between the $P_{1}$ and $P_{2}$ macro solvers, we choose the number of the macro sampling points $N$ as listed in the tables below.

TABLE 4.1. Relative error $\left\|U_{H}-U_{0}\right\|_{H^{1}(D)} /\left\|U_{0}\right\|_{H^{1}(D)}$ and CPU time (in second) of $P_{1}$-vertices vs. $P_{1}$-barycenter for the parameters $\delta=\varepsilon=1 / 100$. $R$ is the CPU time ratio.

\begin{tabular}{rrrrrrr}
\hline \hline & \multicolumn{2}{c}{$P_{1}$-vertices } & \multicolumn{3}{c}{$P_{1}$-barycenter } \\
\hline \hline $\mathrm{N}$ & $\mathrm{M}$ & $\mathrm{H}^{1}$ error & CPU(s) & $\mathrm{H}^{1}$ error & CPU(s) & $\mathrm{R}$ \\
\hline 16 & 4 & $0.40 \mathrm{E}-01$ & 8 & $0.32 \mathrm{E}-01$ & 14 & 1.75 \\
\hline 64 & 8 & $0.11 \mathrm{E}-01$ & 155 & $0.10 \mathrm{E}-01$ & 300 & 1.94 \\
\hline 256 & 16 & $0.26 \mathrm{E}-02$ & 5320 & $0.26 \mathrm{E}-02$ & 10560 & 1.98 \\
\hline
\end{tabular}

TABLE 4.2. Relative error $\left\|U_{H}-U_{0}\right\|_{H^{1}(D)} /\left\|U_{0}\right\|_{H^{1}(D)}$ and $C P U$ time (in second) of $P_{2}-$ edge vs. $P_{2}$-element for the parameters $\delta=\varepsilon=1 / 100 . R$ is the $C P U$ time ratio.

\begin{tabular}{rrrrrrr}
\hline \hline & \multicolumn{2}{c}{$P_{2}$-edge } & \multicolumn{2}{c}{$P_{2}$-element } \\
\hline \hline $\mathrm{N}$ & $\mathrm{M}$ & $\mathrm{H}^{1}$ error & $\mathrm{CPU}(\mathrm{s})$ & $\mathrm{H}^{1}$ error & $\mathrm{CPU}(\mathrm{s})$ & $\mathrm{R}$ \\
\hline 4 & 4 & $0.31 \mathrm{E}-01$ & 1.5 & $0.42 \mathrm{E}-01$ & 2.6 & 1.73 \\
\hline 8 & 8 & $0.11 \mathrm{E}-01$ & 7.6 & $0.10 \mathrm{E}-01$ & 14 & 1.84 \\
\hline 16 & 16 & $0.26 \mathrm{E}-02$ & 64 & $0.27 \mathrm{E}-02$ & 123 & 1.92 \\
\hline
\end{tabular}

It follows from Tables 4.1-4.4 that the cost of $P_{1}$-vertices and $P_{2}$ - edge solvers is approximately one half of the $P_{1}$-barycenter and $P_{2}$-element, respectively. The cost is reduced because the information obtained from the cells located at the common quadrature nodes can be shared. The saving is about one half due to the following simple argument: for a regular triangulation over a general planar bounded domain, the ratio among the total number of vertices, elements and edges is approximately $1: 2: 3$ [26]; see e.g., figure 4.1 .

Figure 4.4 shows the CPU time for different macroscopic solvers when they reach the same accuracy. It is clear that $P_{2}$ solver is more cost-effective than $P_{1}$ solver, i.e., 
TABle 4.3. Relative error $\left\|U_{H}-U_{0}\right\|_{L^{2}(D)} /\left\|U_{0}\right\|_{L^{2}(D)}$ and $C P U$ time (in second) of $P_{1}$-vertices vs. $P_{1}$-barycenter for the parameters $\delta=\varepsilon=1 / 100 . R$ is the CPU time ratio.

\begin{tabular}{crrrrrr}
\hline \hline & \multicolumn{4}{c}{$P_{1}$-vertices } & \multicolumn{2}{c}{$P_{1}$-barycenter } \\
\hline \hline $\mathrm{N}$ & $\mathrm{M}$ & $\mathrm{L}^{2}$ error & CPU(s) & $\mathrm{L}^{2}$ error & CPU(s) & $\mathrm{R}$ \\
\hline 8 & 8 & $0.28 \mathrm{E}-01$ & 3.0 & $0.28 \mathrm{E}-01$ & 4.7 & 1.57 \\
\hline 64 & 64 & $0.47 \mathrm{E}-03$ & 3336 & $0.51 \mathrm{E}-03$ & 6458 & 1.94 \\
\hline
\end{tabular}

TABle 4.4. Relative error $\left\|U_{H}-U_{0}\right\|_{L^{2}(D)} /\left\|U_{0}\right\|_{L^{2}(D)}$ and CPU time (in second) of $P_{2}-$ edge vs. $P_{2}$-element for the parameters $\delta=\varepsilon=1 / 100 . R$ is the CPU time ratio.

\begin{tabular}{rrrrrrr}
\hline \hline & \multicolumn{2}{c}{$P_{2}$-edge } & \multicolumn{2}{c}{$P_{2}$-element } \\
\hline \hline $\mathrm{N}$ & $\mathrm{M}$ & $\mathrm{L}^{2}$ error & $\mathrm{CPU}(\mathrm{s})$ & $\mathrm{L}^{2}$ error & $\mathrm{CPU}(\mathrm{s})$ & $\mathrm{R}$ \\
\hline 4 & 8 & $0.16 \mathrm{E}-01$ & 2.1 & $0.14 \mathrm{E}-01$ & 3.6 & 1.71 \\
\hline 16 & 64 & $0.22 \mathrm{E}-03$ & 631 & $0.20 \mathrm{E}-03$ & 1210 & 1.92 \\
\hline
\end{tabular}

it requires less $\mathrm{CPU}$ time to achieve the same accuracy. We also observe that the advantage of $P_{2}$ solver is more pronounced if the error is measured in the $\mathrm{H}^{1}$ norm. This can be seen from the following simple argument. The total number of numerical integration nodes is almost $N^{2}$ for $P_{1}$-vertices and $3 N$ for $P_{2}$-edge if the error is measured in $\mathrm{H}^{1}$ norm; while the total number of numerical integration nodes changes to $N^{2}$ for $P_{1}$-vertices and $3 N^{4 / 3}$ for $P_{2}$-edge if the error is measured in $\mathrm{L}^{2}$ norm.

REMARK 4.1. It is clear that the higher order macroscopic solver is preferred if the solution $U_{0}$ is smooth enough. However, high order microscopic solver is not preferred since the solution of the cell problem is usually nonsmooth due to the microstructure inside the cell; see Remark 3.11. Therefore, numerical methods tailored to the microstructure are required to achieve better efficiency. We refer to [21] and the references therein for the related discussion on the periodic homogenization problem.

In most of the above examples, we have chosen $\delta=\varepsilon$ and solved the cell problem with periodic boundary conditions. However, this is unrealistic since the exact period $\varepsilon$ is in general unknown due to the measuring error and uncertainty [13]. We turn to the case when $L=\delta / \varepsilon \notin \mathbb{N}$ as advocated in [16, 22], and study the following two-scale periodic homogenization problem that is taken from [22].

EXAMPLE 4.3.

$$
\left\{\begin{aligned}
a(\boldsymbol{x}, \boldsymbol{x} / \varepsilon) & =\left(\frac{1.5+\sin \left(2 \pi x_{1} / \varepsilon\right)}{1.5+\sin \left(2 \pi x_{2} / \varepsilon\right)}+\frac{1.5+\sin \left(2 \pi x_{2} / \varepsilon\right)}{1.5+\cos \left(2 \pi x_{1} / \varepsilon\right)}+\sin \left(4 x_{1}^{2} x_{2}^{2}\right)+1\right) I, \\
f & =10, \quad u=0 \text { on } \partial D
\end{aligned}\right.
$$

where $\varepsilon=1 / 100$. There is no explicit formula of the effective matrix in contrast to (4.6). The homogenized solution $U_{0}$ is computed with a $P_{1}$-vertices solver on a $256 \times 256$ macro mesh. The cell problem $(2.3)$ is solved on a $100 \times 100$ micro mesh with $\delta=\varepsilon$ and periodic boundary condition. We also employ $P_{1}$-vertices solver to compute $U_{H}$, and solve the cell problems (2.3) with a Dirichlet boundary condition. 

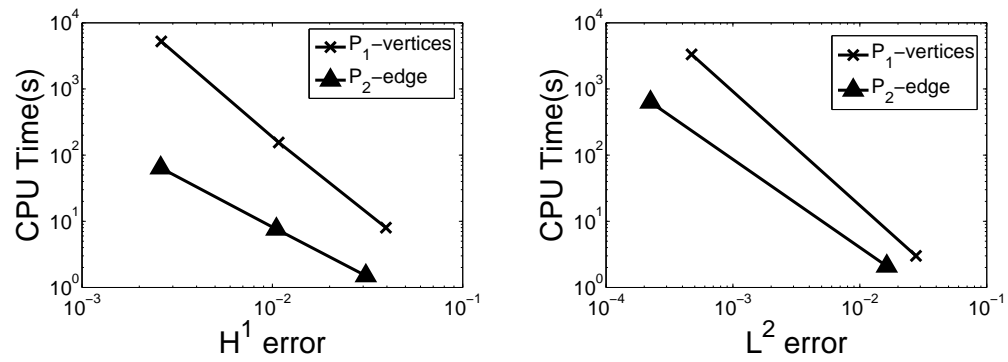

Figure 4.4. CPU time (in seconds) for different solvers at the same accuracy in different relative norm with $\delta=\varepsilon=1 / 100$. Left: $\left\|U_{H}-U_{0}\right\|_{H^{1}(D)} /\left\|U_{0}\right\|_{H^{1}(D)}$; Right: $\| U_{H}-$ $U_{0}\left\|_{L^{2}(D)} /\right\| U_{0} \|_{L^{2}(D)}$.

In both cases, we replace the coefficient $a(\boldsymbol{x}, \boldsymbol{x} / \varepsilon)$ in the cell problem by $a\left(\boldsymbol{x}_{\ell}, \boldsymbol{x} / \varepsilon\right)$. The estimates (4.1) and (4.2) change to

$$
\begin{aligned}
\left\|U_{0}-U_{H}\right\|_{H^{1}(D)} & \leq C\left(N^{-1}+L^{-1}+L^{2} M^{-2}\right), \\
\left\|U_{0}-U_{H}\right\|_{L^{2}(D)} & \leq C\left(N^{-2}+L^{-1}+L^{2} M^{-2}\right),
\end{aligned}
$$

which suggest the following strategy of refining the macro-micro meshes and a criterion for determining the size of the microcell:

$$
\left\{\begin{array}{l}
L=N, \quad M=N^{3 / 2}, \quad H^{1} \text { error }, \\
L=N^{2}, \quad M=N^{3}, \quad L^{2} \text { error. }
\end{array}\right.
$$

The refinement strategy is understood as follows: in terms of $\mathrm{H}^{1}$ error, the microcell size is doubly enlarged, the macro-micro meshes are doubly and triply $(2 \sqrt{2})$ refined, respectively; in terms of $\mathrm{L}^{2}$ error, the microcell size is four times enlarged and the macro-micro meshes are refined to the ratio of 2 and 8 , respectively. This seems the best refinement strategy for the optimal convergence rate with minimal computational cost, since the error estimates (4.1) and (4.2) are sharp. We summarize the results in Tables 4.5 and 4.6 (in the first three rows), which clearly illustrate the first and second order of convergence for the relative $\mathrm{H}^{1}$ and $\mathrm{L}^{2}$ error, respectively.

TABLE 4.5. Relative error $\left\|U_{H}-U_{0}\right\|_{H^{1}(D)} /\left\|U_{0}\right\|_{H^{1}(D)}$ with $P_{1}$-vertices solver for $\varepsilon=1 / 100$ when macro, micro sampling points $N, M$ are increased and the microcell size $\delta$ is enlarged simultaneously.

\begin{tabular}{crrrr}
\hline \hline $\mathrm{N}$ & $\delta / \varepsilon$ & $\mathrm{M}$ & $\mathrm{H}^{1}$ error & order \\
\hline 4 & $100 / 24$ & 8 & $0.58 \mathrm{E}-01$ & \\
\hline 8 & $100 / 12$ & 25 & $0.31 \mathrm{E}-01$ & 0.90 \\
\hline 16 & $100 / 6$ & 64 & $0.16 \mathrm{E}-01$ & 0.95 \\
\hline \hline 4 & $100 / 64$ & 4 & $0.60 \mathrm{E}-01$ & \\
\hline 8 & $100 / 32$ & 10 & $0.33 \mathrm{E}-01$ & 0.86 \\
\hline 16 & $100 / 16$ & 25 & $0.15 \mathrm{E}-01$ & 1.14 \\
\hline
\end{tabular}

In view of the results in the above tables, the error is even decreased when the cell size is larger than the macro element size as predicted by the theoretical results in Theorems 3.2 and 3.3 ; see figure 4.5 . 
TABLE 4.6. Relative error $\left\|U_{H}-U_{0}\right\|_{L^{2}(D)} /\left\|U_{0}\right\|_{L^{2}(D)}$ with $P_{1}$-vertices solver for $\varepsilon=1 / 100$ when macro, micro sampling points $N, M$ are increased and the microcell size $\delta$ is enlarged simultaneously.

\begin{tabular}{rrrrr}
\hline \hline $\mathrm{N}$ & $\delta / \varepsilon$ & $\mathrm{M}$ & $\mathrm{L}^{2}$ error & order \\
\hline 2 & $100 / 24$ & 8 & $0.11 \mathrm{E}-00$ & \\
\hline 4 & $100 / 6$ & 64 & $0.37 \mathrm{E}-01$ & 1.57 \\
\hline 8 & $100 / 1.55$ & 512 & $0.10 \mathrm{E}-01$ & 1.89 \\
\hline \hline 2 & $100 / 64$ & 4 & $0.13 \mathrm{E}-00$ & \\
\hline 4 & $100 / 16$ & 32 & $0.46 \mathrm{E}-01$ & 1.50 \\
\hline 8 & $100 / 4$ & 256 & $0.14 \mathrm{E}-01$ & 1.72 \\
\hline
\end{tabular}

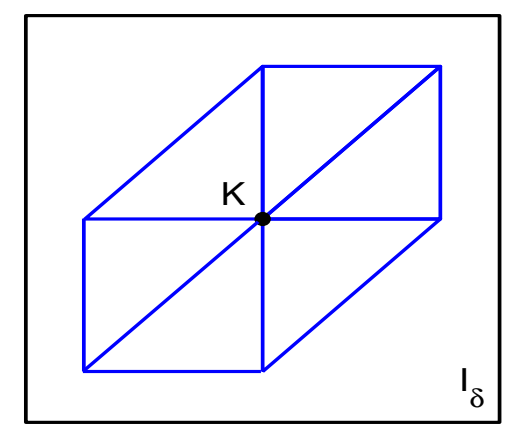

Figure 4.5. Showcase of the microcell used in Example 4.3. It is larger than the macro element.

It was pointed out in [18] that the constant in front of the resonance error $\mathcal{O}\left(L^{-1}\right)$ is much smaller than the constants in front of other terms in the error bound. The same phenomenon was observed in HMM-FEM. Therefore, we may begin with a smaller microcell in the refinement strategy (4.9). It follows from the last three lines of Tables 4.5 and 4.6 that the same order of convergence can be retained even with a much smaller $L$ and $M$. Therefore, the cost is even reduced.

Let us compare the mesh refinement strategies for the different choices of cell size. If we consider the $\mathrm{H}^{1}$ error and $\delta=\varepsilon$, then the microcell should be refined to the ratio of $\sqrt{2}$ to achieve the optimal accuracy according to (4.7). However, for the general case when $\delta / \varepsilon \notin \mathbb{N}$, the microcell should be almost triply refined. Otherwise, the microstructures inside each cell has not been fully resolved and the optimal convergence rate can not be guaranteed. The same scenario happens for the $\mathrm{L}^{2}$ error.

Now we come to the global phase of the accuracy of HMM-FEM. In all examples studied, we fixed the slow variable of the coefficients to solve the cell problem, namely, the coefficient $a(\boldsymbol{x}, \boldsymbol{x} / \varepsilon)$ is replaced by $a\left(\boldsymbol{x}_{\ell}, \boldsymbol{x} / \varepsilon\right)$. Therefore, the term $\delta$ disappears from $e(\mathrm{HMM})$. This is not the case in general. If the term $\mathcal{O}(\delta)$ appears in $e(\mathrm{HMM})$, then the overall accuracy threshold is of $\mathcal{O}(\sqrt{\varepsilon})$. The strategy (4.9) can still be used to refine the macro-micro meshes under this threshold. In the future, we will develop methods to improve the order of the term $\mathcal{O}(\delta)$, which may be achieved by employing certain special average methods in the evaluation of the effective matrix; see e.g., [15]. 


\section{Conclusion and discussion}

We have proposed two new numerical integration schemes in the framework of HMM-FEM. Compared with the numerical integration schemes commonly used in HMM-FEM, the proposed methods significantly reduce the cost while retaining the accuracy. Optimal error estimates are proven, which generalize the previous estimates to the non-self-adjoint elliptic problems. The technique developed in this paper can be employed to obtain improved error estimates for the parabolic homogenization problems [23]. Our sharp estimates also give a natural refinement strategy for the microscopic and macroscopic solvers. Based on this refinement strategy, extensive numerical experiments are performed, and the results are in good agreement with the theoretical prediction.

Future work will involve the extension of the new numerical integration schemes to three-dimensional problems in which the computational cost saving is more pronounced. Equally interesting problem is to study the random homogenization problems. The first step is to prove the sharp bound for $e$ (HMM), which is highly nontrivial. The development and analysis of the new numerical integration schemes for HMM-DG is also promising.

Acknowledgement. The authors are grateful to professors W. E and X.-Y. Yue for helpful discussions.

\section{Appendix A. Proof of Lemma 3.9.}

Proof of (3.20).

In case of $m=0$, the estimate (3.20) can be obtained in the same manner as the estimate (3.3) for $w_{h}^{\varepsilon}$.

Define $w_{1}^{\varepsilon} \equiv \widehat{w}^{\varepsilon}-W_{\ell}$. It is clear that $w_{1}^{\varepsilon}$ satisfies

$$
\left\{\begin{aligned}
\mathcal{L}\left(w_{1}^{\varepsilon}\right)=F \quad & \text { in } \quad I_{\delta}, \\
w_{1}^{\varepsilon}=0 & \text { on } \quad \partial I_{\delta},
\end{aligned}\right.
$$

where

$$
\mathcal{L}\left(w_{1}^{\varepsilon}\right)=-\left(a_{\ell}^{\varepsilon}\right)_{i j} \frac{\partial^{2} w_{1}^{\varepsilon}}{\partial x_{i} \partial x_{j}} \quad \text { and } \quad F=\frac{\partial\left(a_{\ell}^{\varepsilon}\right)_{i j}}{\partial x_{i}} \frac{\partial \widehat{w}^{\varepsilon}}{\partial x_{j}} .
$$

Integrating by parts, we obtain

$$
\begin{aligned}
\int_{I_{\delta}}\left|\mathcal{L}\left(w_{1}^{\varepsilon}\right)\right|^{2} \mathrm{~d} \boldsymbol{x}= & \int_{I_{\delta}}\left(a_{\ell}^{\varepsilon}\right)_{i j}\left(a_{\ell}^{\varepsilon}\right)_{k l} \frac{\partial^{2} w_{1}^{\varepsilon}}{\partial x_{i} \partial x_{j}} \frac{\partial^{2} w_{1}^{\varepsilon}}{\partial x_{k} \partial x_{l}} \mathrm{~d} \boldsymbol{x} \\
= & \int_{I_{\delta}}\left(a_{\ell}^{\varepsilon}\right)_{i j}\left(a_{\ell}^{\varepsilon}\right)_{k l} \frac{\partial^{2} w_{1}^{\varepsilon}}{\partial x_{i} \partial x_{k}} \frac{\partial^{2} w_{1}^{\varepsilon}}{\partial x_{j} \partial x_{l}} \mathrm{~d} \boldsymbol{x} \\
& -\int_{I_{\delta}} \frac{\partial}{\partial x_{j}}\left(\left(a_{\ell}^{\varepsilon}\right)_{i j}\left(a_{\ell}^{\varepsilon}\right)_{k l}\right) \frac{\partial w_{1}^{\varepsilon}}{\partial x_{i}} \frac{\partial^{2} w_{1}^{\varepsilon}}{\partial x_{k} \partial x_{l}} \mathrm{~d} \boldsymbol{x} \\
& +\int_{I_{\delta}} \frac{\partial}{\partial x_{k}}\left(\left(a_{\ell}^{\varepsilon}\right)_{i j}\left(a_{\ell}^{\varepsilon}\right)_{k l}\right) \frac{\partial w_{1}^{\varepsilon}}{\partial x_{i}} \frac{\partial^{2} w_{1}^{\varepsilon}}{\partial x_{j} \partial x_{l}} \mathrm{~d} \boldsymbol{x}+\int_{\partial I_{\delta}} I(s) \mathrm{d} \sigma,
\end{aligned}
$$

where the line integrand $I(s)$ is defined as

$$
I(s) \equiv\left(a_{\ell}^{\varepsilon}\right)_{i j}\left(a_{\ell}^{\varepsilon}\right)_{k l} \frac{\partial w_{1}^{\varepsilon}}{\partial x_{i}}\left[\frac{\partial^{2} w_{1}^{\varepsilon}}{\partial x_{k} \partial x_{l}} n_{j}-\frac{\partial^{2} w_{1}^{\varepsilon}}{\partial x_{j} \partial x_{l}} n_{k}\right],
$$


where $n_{j}$ is the $j$-th component of the outward unit normal of $\partial I_{\delta}$. By [19, p. 151] and the fact that $I_{\delta}$ is convex, we have

$$
\int_{\partial I_{\delta}} I(s) \mathrm{d} \sigma \geq 0 .
$$

This together with the ellipticity of $a_{\ell}^{\varepsilon}$ leads to

$$
\begin{aligned}
\int_{I_{\delta}}\left|\mathcal{L}\left(w_{1}^{\varepsilon}\right)\right|^{2} \mathrm{~d} \boldsymbol{x} & \geq \lambda^{2} \int_{I_{\delta}}\left|D^{2} w_{1}^{\varepsilon}\right|^{2} \mathrm{~d} \boldsymbol{x}-2 \Lambda\left\|\nabla a_{\ell}^{\varepsilon}\right\|_{L^{\infty}(D)} \int_{I_{\delta}}\left|\nabla w_{1}^{\varepsilon} D^{2} w_{1}^{\varepsilon}\right| \mathrm{d} \boldsymbol{x} \\
& \geq \frac{\lambda^{2}}{2} \int_{I_{\delta}}\left|D^{2} w_{1}^{\varepsilon}\right|^{2} \mathrm{~d} \boldsymbol{x}-\frac{2 \Lambda^{2}}{\lambda^{2}}\left\|\nabla a_{\ell}^{\varepsilon}\right\|_{L^{\infty}(D)}^{2} \int_{I_{\delta}}\left|\nabla w_{1}^{\varepsilon}\right|^{2} \mathrm{~d} \boldsymbol{x} .
\end{aligned}
$$

It follows from (A.1) that

$$
\begin{aligned}
\left\|D^{2} w_{1}^{\varepsilon}\right\|_{L^{2}\left(I_{\delta}\right)}^{2} & \leq \frac{4 \Lambda^{2}}{\lambda^{4}}\left\|\nabla a_{\ell}^{\varepsilon}\right\|_{L^{\infty}(D)}^{2}\left\|\nabla w_{1}^{\varepsilon}\right\|_{L^{2}\left(I_{\delta}\right)}^{2}+\frac{2}{\lambda^{2}}\|F\|_{L^{2}\left(I_{\delta}\right)}^{2} \\
& \leq \frac{4 \Lambda^{2}}{\lambda^{4}}\left\|\nabla a_{\ell}^{\varepsilon}\right\|_{L^{\infty}(D)}^{2}\left\|\nabla w_{1}^{\varepsilon}\right\|_{L^{2}\left(I_{\delta}\right)}^{2}+\frac{2}{\lambda^{2}}\left\|\nabla a_{\ell}^{\varepsilon}\right\|_{L^{\infty}(D)}^{2}\left\|\nabla \widehat{w}^{\varepsilon}\right\|_{L^{2}\left(I_{\delta}\right)}^{2} \\
& \leq \frac{\Lambda^{2}}{\lambda^{4}}\left\|\nabla a_{\ell}^{\varepsilon}\right\|_{L^{\infty}(D)}^{2}\left(4\left\|\nabla w_{1}^{\varepsilon}\right\|_{L^{2}\left(I_{\delta}\right)}^{2}+2\left\|\nabla W_{\ell}\right\|_{L^{2}\left(I_{\delta}\right)}^{2}\right)
\end{aligned}
$$

Taking $z=w_{1}^{\varepsilon}$ in (3.7), we obtain

$$
\int_{I_{\delta}\left(\boldsymbol{x}_{\ell}\right)} \nabla w_{1}^{\varepsilon} \cdot a_{\ell}^{\varepsilon} \nabla w_{1}^{\varepsilon} \mathrm{d} \boldsymbol{x}=-\int_{I_{\delta}\left(\boldsymbol{x}_{\ell}\right)} \nabla w_{1}^{\varepsilon} \cdot a_{\ell}^{\varepsilon} \nabla W_{\ell} \mathrm{d} \boldsymbol{x}
$$

which implies

$$
\left\|\nabla w_{1}^{\varepsilon}\right\|_{L^{2}\left(I_{\delta}\right)} \leq \frac{\Lambda}{\lambda}\left\|\nabla W_{\ell}\right\|_{L^{2}\left(I_{\delta}\right)} .
$$

Substituting the above inequality into (A.2), we obtain

$$
\left\|D^{2} w_{1}^{\varepsilon}\right\|_{L^{2}\left(I_{\delta}\right)}^{2} \leq\left(2 \Lambda^{2} / \lambda^{4}+4 \Lambda^{4} / \lambda^{6}\right)\left\|\nabla a_{\ell}^{\varepsilon}\right\|_{L^{\infty}(D)}^{2}\left\|\nabla W_{\ell}\right\|_{L^{2}\left(I_{\delta}\right)}^{2} .
$$

Using the fact that $\left\|\nabla a_{\ell}^{\varepsilon}\right\|_{L^{\infty}(D)} \leq C / \varepsilon$, we obtain

$$
\left\|D^{2} w_{1}^{\varepsilon}\right\|_{L^{2}\left(I_{\delta}\right)} \leq C \varepsilon^{-1}\left\|\nabla W_{\ell}\right\|_{L^{2}\left(I_{\delta}\right)},
$$

where $C$ only depends on $\lambda, \Lambda$ and $\left\|a\left(\boldsymbol{x}_{\ell}, \boldsymbol{y}\right)\right\|_{C^{1}(Y)}$. Therefore we obtain (3.20) with $m=1$.

\section{REFERENCES}

[1] A. Abdulle, On a priori error analysis of fully discrete heterogeneous multiscale FEM, Multiscale Model. Simul., 4, 447-459, 2005.

[2] A. Abdulle, The finite element heterogeneous multiscale method: a computational strategy for multiscale PDEs, GAKUTO Internat. Ser. Math. Sci. Appl., 31, 133-181, 2009.

[3] A. Abdulle and A. Nonnenmacher, A short and versatile finite element multiscale code for homogenizaton problems, Comput. Methods Appl. Mech. Engrg., 198, 2839-2859, 2009.

[4] A. Abdulle and B. Engquist, Finite element heterogeneous multiscale methods with near optimal computational complexity, Multiscale Model. Simul., 6, 1059-1084, 2007. 
[5] A. Abdulle and C. Schwab, Heterogeneous multiscale FEM for diffusion problem on rough surfaces, Multiscale Model. Simul., 3, 195-220, 2005.

[6] R.A. Adams and J.J.F. Fournier, Sobolev Spaces, 2nd ed., Academic Press, New York, 2003.

[7] I. Babuška, B. Andersson, P. Smith and K. Levin, Damage analysis of fiber composites. I. statistical analysis on fiber scale, Comput. Methods Appl. Mech. Engrg., 172, 27-77, 1999.

[8] I. Babuška, F. Nobile and R. Tempone, Worst case scenario analysis for elliptic problems with uncertainty, Numer. Math., 101, 185-219, 2005.

[9] A. Bensoussan, J.L. Lions and G.C. Papanicolaou, Asymptotic Analysis for Periodic Structures, North-Holland, Amsterdam, 1978.

[10] S. Chen, W. E and C.-W. Shu, The heterogeneous multiscale method based on the discontinuous Galerkin method for hyperbolic and parabolic problems, Multiscale Model. Simul., 3, 871894, 2005.

[11] P.G. Ciarlet, The Finite Element Method for Elliptic Problems, North-Holland, Amsterdam, 1978.

[12] D. Cioranescu and P. Donato, An Introduction to Homogenization, Oxford University Press, New York, 1999.

[13] B. DeVolder, J. Glimm, J.W. Grove, Y. Kang, Y. Lee, K. Pao, D.H. Sharp and K. Ye, Uncertainty quantification for multiscale simulations, J. Fluids Eng., 124, 29-41, 2002.

[14] R. Du and P.B. Ming, Convergence of heterogeneous multiscale method for composite material, preprint, 2009.

[15] W. E and B. Engquist, The heterogeneous multiscale methods, Commun. Math. Sci., 1, 87-132, 2003.

[16] W. E, P.B. Ming and P.W. Zhang, Analysis of the heterogeneous multiscale method for elliptic homogenization problems, J. Amer. Math. Soc., 18, 121-156, 2005.

[17] P.C. Hammer and A.H. Stroud, Numerical integration over simplexes, Math. Tables Other Aids to Comput., 10, 137-139, 1956.

[18] T.Y. Hou and X.-H. Wu, A multiscale finite element method for elliptic problems in composite materials and porous media, J. Comput. Phys., 134, 169-189, 1997.

[19] O.A. Ladyzhenskaya and N.N. Ural'tseva, Linear and Quasi-linear Elliptic Equations, Nauka, Moscow, 1964, in Russian; English translation: Academic Press, New York, 1968; 2nd Russian ed. 1973.

[20] Y.Y. Li and M. Vogelius, Gradient estimates for solutions to divergence form elliptic equations with discontinuous coefficients, Arch. Rational Mech. Anal., 153, 91-151, 2000.

[21] A.M. Matache and C. Schwab, Two-scale FEM for homogenization problems, M2AN Math. Model. Numer. Anal., 36, 537-572, 2002.

[22] P.B. Ming and X.Y. Yue, Numerical methods for multiscale elliptic problems, J. Comput. Phys., $214,421-445,2006$.

[23] P.B. Ming and P.W. Zhang, Analysis of the heterogeneous multiscale method for parabolic homogenization problems, Math. Comput., 76, 153-177, 2007.

[24] MUMPS: a parallele sparse direct solver: http://mumps.enseeiht.fr/.

[25] S. Pecullan, L.V. Gibiansky and S. Torquato, Scale effects on the elastic behavior of periodic and hierarchical two-dimensional composites, J. Mech. Phys. Solids, 47, 1509-1542, 1999.

[26] G. Strang and G.J. Fix, An Analysis of the Finite Element Method, Prentice-Hall, Englewood Cliffs, N.J., 1973.

[27] L. Tartar, H-Convergence, Course Peccot, Collége de France, March 1977. Partially written by F. Murat. Séminaire d'Analyse Fonctionnelle et Numérique de l'Université d'Alger, 1977-1978. English Translation: F. Murat and L. Tartar, H-Convergence, in Topics in the Mathematical Modeling of Composite Materials, A. Cherkaev and R. Kohn (eds.), Birkhäuser, Boston, 21-43, 1997.

[28] X.Y. Yue and W. E, The local microscale problem in the multiscale modeling of strongly heterogeneous media: effects of boundary conditions and cell size, J. Comput. Phys., 222, 556-572, 2007. 